\title{
Modélisation d'un système lotique pollué par une charge organique : prise en compte de l'activité des microorganismes benthiques
}

\section{Modelisation of a Iotic ecosystem polluted by a pigstywaste: dynamic of dissolved carbon and benthic microorganism}

\author{
B. Cazelles et D. Fontvieille
}

Volume 2, numéro 4, 1989

URI : https://id.erudit.org/iderudit/705041ar

DOI : https://doi.org/10.7202/705041ar

\section{Aller au sommaire du numéro}

\section{Éditeur(s)}

Université du Québec - INRS-Eau, Terre et Environnement (INRS-ETE)

ISSN

0992-7158 (imprimé)

1718-8598 (numérique)

\section{Découvrir la revue}

Citer cet article

Cazelles, B. \& Fontvieille, D. (1989). Modélisation d'un système lotique pollué par une charge organique : prise en compte de l'activité des microorganismes benthiques. Revue des sciences de l'eau / Journal of Water Science, 2(4), 511-541. https://doi.org/10.7202/705041ar

\section{Résumé de l'article}

Les études entreprises depuis 1982 sur un petit cours d'eau pollué par une forte charge organique, ont servi de base au développement d'un modèle mathématique destiné à appréhender le fonctionnement des mécanismes d'auto-épuration.

L'écriture du modèle a débuté par la description de l'écoulement du cours d'eau et des mécanismes de transports associés. Deux sous-modèles ont été utilisés dans ce but : un sous-modèle hydrodynamique dérivé du modèle de Saint-Venant et un sous-modèle de transport, constitué par l'équation classique de convection-dispersion. Ces deux sous-modèles sont appliqués à un écoulement monodimensionnel, non uniforme et non stationnaire.

Dans les petits cours d'eau le compartiment benthique joue un rôle prépondérant du point de vue de l'ensemble des processus détritiques. Le modèle a donc été appliqué, dans un premier temps, à la simulation de la dynamique du carbone organique dissous et des microorganismes benthiques. Les mécanismes de biodégradation sont modélisés, ici, en utilisant l'analogie entre les processus détritiques et l'activité des biomasses des procédés de traitement des eaux usées. Le modèle de biofilm développé permet, par sa structure, d'englober tous les mécanismes physiques et biologiques qui entrent dans les phénomènes de disparition et de dégradation de la matière organique dissoute.

Ce modèle est utilisé, sous sa forme actuelle, en tant qu'outil cognitif destiné à accroître la connaissance des mécanismes d'auto-épuration de la matière organique dissoute dans les petits cours d'eau. 


\section{Modélisation d'un système lotique pollué par une charge organique : prise en compte de l'activité des microorganismes benthiques}

Modelisation of a lotic ecosystem polluted by a pigstywaste : dynamic of dissolved carbon and benthic microorganism

Les études entreprises depuis 1982 sur un petit cours d'eau pollué par une forte charge organique, ont servi de base au développement $d$ 'un modèle mathématique destinè à appréhender te fonctionnement des mécanismes d'auto-épuration.

L'écriture du modèle a débuté par ta description de l'écoulement du cours d'eau et des mécanismes de transports associés. Deux sous-modèles ont été utilisés dans ce but : un sousmodèle hydrodynamique dérivé du modèle de Saint-Venant et un sous-modèle de transport, constitué par l'équation classique de convection-dispersion. Ces deux sous-modèles sont appliqués à un écoulement monodimensionnel, non uniforme et non stationnaire.

Dans les petits cours d'eau le compartiment benthique joue un rôle prépondérant du point de vue de l'ensemble des processus détritiques. Le modèle a donc été appliqué, dans un premier temps, à la simulation de la dynamique du carbone organique dissous et des microorganismes benthiques.

(1) Anjou-Recherche, B.P. 76, 78600 Maisons-Lafitte, détaché à l'Université de Savoie, France.

(2) Laboratoire d’Ecologie, Université de Savoie, B.P. 1104, 73011 Chambéry, France. 
Les mécanismes de biodégradation sont modélisés, ici, en utilisant l'analogie entre les processus détritiques et l'activité des biomasses des procédés de traitement des eaux usées. Le modèle de biofilm développé permet, par sa structure, d'englober tous les mécanismes physiques et biologiques qui entrent dans les phénomènes de disparition et de dégradation de la matière organique dissoute.

Ce modèle est utilisé, solis sa forme actuelle, en tant qu'outil cognitif destiné à accroître la connaissance des mécanismes d'auto-épuration de la matière organique dissoute dans les petits cours d'eau.

Mots clés : Cours d'eau, modèle mathématique, simulation, équation de convection-dispersion, auto-épuration, microorganismes, biofilm.

Several studies have been performed since 1982 on a small organically polluted stream. Their results have been used to conceive a mathematical model with the purpose of simulating the self-purifieation mechanisms.

The physical part of this model includes a hydrodynamic component derived from Saint-Venant's equations which is coupled with a transport model based on the classical convectiondispersion equation. The model is applied to unidirectional, non-uniform, unsteady flow conditions.

As the main detritic processes which occur in small streams take place in the sediment, the model has been applied, first, to simulate the dynamics of both dissolved organic matter and benthic microorganisms.

The simulation of the biodegradation mechanisms is based, in this work, on the analogy between the detritic processes in streams and the activity of the microbial biomass in wastewater treatment plants. From the structure of the biofilm model proposed, it was assessed that nearly all mechanisms involved in the removal and degradation of dissolved organic matter may be considered.

In these studies, the model is used as a cognitive tool intended to increase the understanding of self-purification mechanisms applied to dissolved organic matter is small streams.

Key-words : Stream, mathematical model, simulation, convectiondispersion equation, self-purification, microorganisms, biofilm. 
La pollution organique des eaux se traduit, notamment, par un abaissement de la richesse écologique des peuplements accompagné d'une prolifération d'espèces adaptëes aux nouvelles conditions environnementales. Des mécanismes dits d'auto-épuration interviennent alors pour êliminer progressivement la charge polluante et pour redonner au cours d'eau un état proche de l'équilibre original. Ces mécanismes ont une triple nature (WUHRMANN, 1972) :

- physique (dilution, adsorption, sëdimentation...) ;

- chimique (oxydations ...) ;

- biochimique et biologique (assimilation, respiration, ...).

Pour appréhender le rôle et l'importance de ces mécanismes d'autoépuration, ainsi que leur ordre de grandeur et les liens qui les unissent, deux campagnes de mesures (septembre 1981 - avril 1982, novembre 1982 - mars 1984) ont été conduites successivement sur un petit cours d'eau savoyard, 1 'Albenche.

Le besoin d'outils d'aide à la comprêhension permettant une synthèse fonctionnelle des multiples connaissances ponctuelles acquises dans divers domaines de l'écologie, de même que la nécessité de disposer, pour des etudes d'impacts, d'un outil au moins grossièrement prévisionnel des effets écologiques à attendre, sont autant de raisons qui ont conduit à la réalisation et a 1 'utilisation de plus en plus fréquentes de modèles mathématiques. C'est le cas en particulier des travaux touchant à l'étude et à la gestion de la qualité des eaux dans lesquels de très nombreux modèles sont proposés.

On peut définir ces modèles comme des quasi analogues mathématiques du comportement des systèmes réels. En synthëtisant et en intégrant, les connaissances acquises sur un écosystème, leur construction permet de structurer les concepts sous une forme non ambiguē de type mathématique (GENTIL, 1982 ; BECK, 1983).

Les simulations numériques effectuées à l'aide de ces modèles, donnent la possibilité de calculer l'évolution spatiale et/ou temporelle d'un certain nombre de variables caractéristiques de l'écosystème étudié. Ces simulations apportent une vision analytique et mécaniste du système. Elles permettent notamment, de tester différentes hypothèses sur sa structure et son fonctionnement, ainsi que sur la coherence du savoir acquis, par l'introduction simultanée de diffërentes hypothèses dans le modẻle (O'NEIL et $a l ., 1979 b$; Mac INTIRE, 1983).

Ces techniques de modélisation ont été mises en oeuvre dans le cadre des études entreprises sur 1'Albenche. Elles ont abouti a l'elaboration d'un modèle mathẻmatique de ce système écologique qui permet 1'étude qualitative et quantitative d'un certain nombre de processus participant à I'auto-épuration.

La partie physique du modèle a été décrite dans un précédent article (CAZELLES et FONTVIEILIE, 1989). Dans ce travail, selils sont décrits 
la partie biologique du modẻle et certains résultats obtenus lors de la simulation de la dynamique du carbone organique dissous et des microorganismes benthiques.

L'Albenche est un petit cours d'eau de basse montagne situé sur le versant Est du Massif de la Chambotte (cartes I.G.N. Rumilly 5-6, 7-8, 1 : 25000), dans le bassin versant du lac du Bourget. Il alimente la Deisse, affluent du Sierroz qui se jette dans le lac du Bourget au niveau d'Aix-les-Bains.

Le bassin versant de l'Albenche est essentiellement agricole. Ce cours d'eau reçoit jusqu'au lieu-dit la Croix du Sable, différents rejets domestiques, provenant de hameaux ou d'habitations isolées, et des rejets agricoles, représentés surtout par les eaux de lavage d'une porcherie. En aval de ce point, l'Albenche prēsente tous les stades d'une auto-épuration menée jusqu'à un état de restauration quasi complet à partir du village d'Albens.

L'étude porte sur le tronçon de I'Albenche situé entre le rejet de Ia porcherie et Albens (figure 1). Ce tronçon présente l'avantage d'être un milieu relativement simple, aux dimensions réduites, et dont le seul affluent, situé en amont d'Albens, est de faible importance. Quatre stations d'étude, correspondant à différents stades de 1 'auto-épuration, $y$ ont été définies :

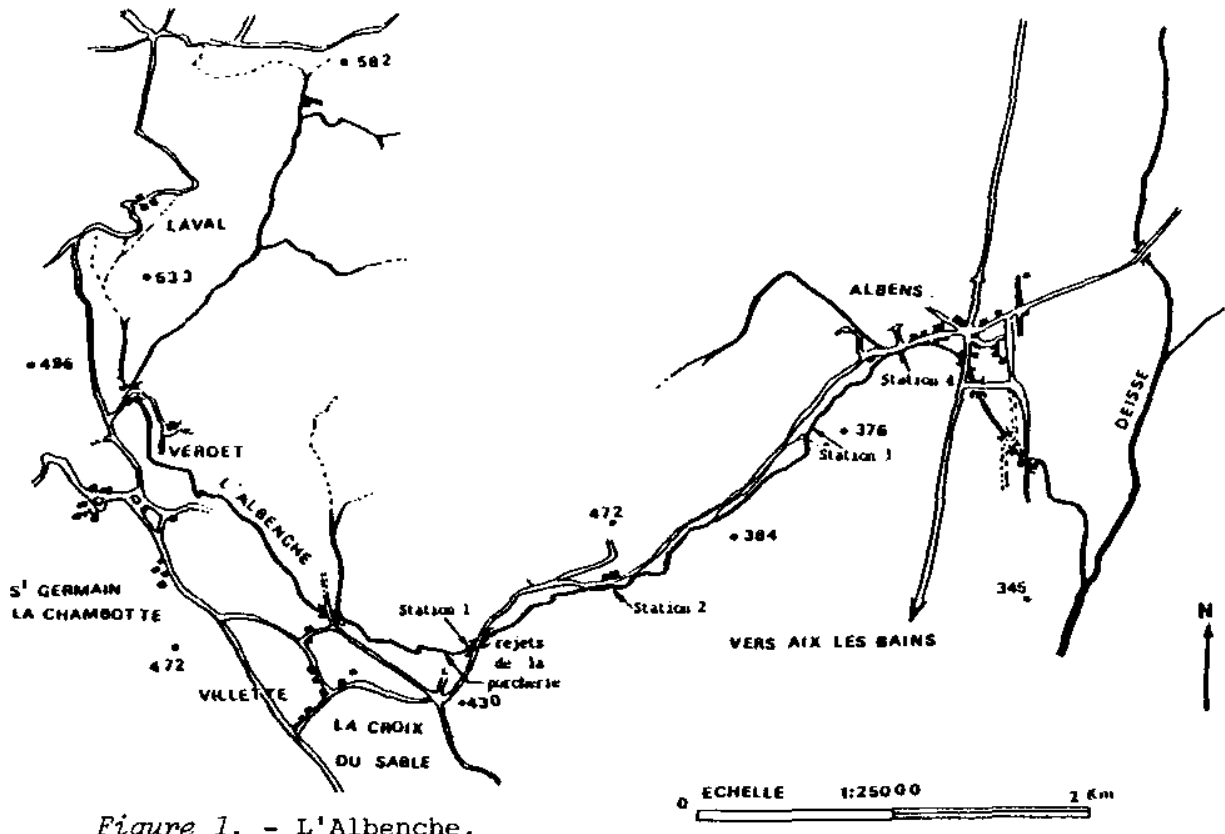

Figure 1. - L'Albenche.

Figure 1. - The Albenche river. 
- la station 1 située à $150 \mathrm{~m}$ du rejet dans une zone polysaprobe ;

- la station 2 située à $1000 \mathrm{~m}$ du rejet ;

- la station 3 située à $2400 \mathrm{~m}$ du rejet ;

- la station 4 située à $3050 \mathrm{~m}$ du rejet dans une zone où le cours a'eau commence à retrouver sa qualité initiale.

Certaines caractéristiques mésologiques et morphodynamiques de ces stations sont résumées dans le tableau 1.

Tableau 1. - Caractéristiques mésologiques et morphodynamique de l'Albenche.

Table 1. - Mesologic and morphodynamic characteristics of the Albenche river.

\begin{tabular}{|c|c|}
\hline Stations & 2 \\
\hline $\begin{array}{l}\text { Longueur } \\
\text { totale }\end{array}$ & $11.5 \mathrm{~km}$ \\
\hline $\begin{array}{l}\text { Longueur } \\
\text { étudiée }\end{array}$ & $3.2 \mathrm{~km}$ \\
\hline $\begin{array}{l}\text { Bassin } \\
\text { versant }\end{array}$ & $\mathrm{km}^{2}$ \\
\hline $\begin{array}{l}\text { Pente } \\
\text { moyenne }\end{array}$ & du cours d'eau : $1.48 ;$ du tronçon étudié : 2 \& \\
\hline $\begin{array}{l}\text { Gamune de } \\
\text { débit }\end{array}$ & 15 à $60001 . s^{-1}$ \\
\hline $\begin{array}{l}\text { Gamme de } \\
\text { température }\end{array}$ & I à $18^{\circ} \mathrm{C}$ \\
\hline Coordonnèes & 879.05 \\
\hline Lambert & 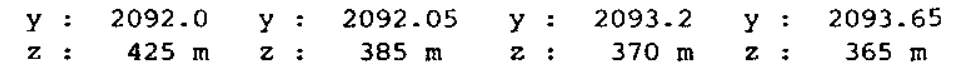 \\
\hline Largeur & $\begin{array}{l}2 \text { a } 5 \mathrm{~m} \text { suivant les faciès et les saisons; } 2.5 \mathrm{~m} \text { en } \\
\text { moyenne }\end{array}$ \\
\hline Profondeur & $\begin{array}{l}5 \text { à } 80 \mathrm{~cm} \text { selon les faciès et les saisons ; } 20 \mathrm{~cm} \text { en } \\
\text { moyenne }\end{array}$ \\
\hline $\begin{array}{l}\text { Distance } \\
\text { rejet-station } \\
\text { Oxygène } \\
\text { dissous }\end{array}$ & 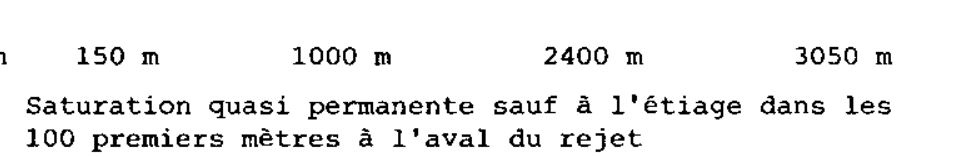 \\
\hline $\begin{array}{l}\text { Couverture } \\
\text { du fond du } \\
\text { ruisseau }\end{array}$ & $\begin{array}{l}\text { Cormunautés de bactéries filamenteuses principalement } \\
\text { Sphaerotilus sp. et Beggiatoa sp. } \\
\begin{array}{l}\text { Plus ou moins Présente selon Développement plus ou moins } \\
\text { abondante } \\
\text { toute l'année }\end{array}\end{array}$ \\
\hline $\begin{array}{l}\text { Couverture } \\
\text { végétale } \\
\text { des berges }\end{array}$ & Couvert végétal dense : 95 \& \\
\hline
\end{tabular}




\section{3 - analyse du système "albenche"}

Dans les petits cours d'eau, tels que l'Albenche, la biomasse fixée, joue un rôle prépondérant dans les processus de dégradation de la matière organique (GEESEY et al., 1978, SRINANTHAXUMAR et AMIRTHARAJAH, 1983). Ces processus de dégradation sont localisés préférentiellement dans le compartiment benthique comme l'ont souligné de nombreux auteurs (KARLSTROM, 1978 ; LOCK, 1981 ; VANDER BORGHT et al., 1981 ; HARREMOES, 1982 ; HVITVED-JACOBSEN, 1982 ; BOYLE et SCOTT, 1984 ; LOCK et al., 1984 ; HICKEY, 1988). Les mesures effectuées in situ, dans la veine liquide, dans 1 'Albenche, ont confirmée cette prépondérance du compartiment benthique. L'activité biologique mesurêe par la production nette de $\mathrm{CO}_{2}$ (FONTVIEILLE et RENAUD, 1982) s'est révélée $y$ être inférieure au seuil de détection de la méthode utilisée.

L'interprētation des campagnes de mesures (FONTVIEIILE et CAZELLES, 1985 ; FONTVIEILLE et CAZELLES, 1988) complétée par une première étude bibliographique (BOLING et al., 1975 ; CLESCERI et al., 1977 ; Mac INTIRE et COLBY, 1978 ; O'NEIL et al., 1979a ; WEBSTER et al., 1979 ; THERIEN et al., 1981 ; NEWBOLD et al., 1983 ; WEBSTER, 1983) ont conduit à l'ëcriture d'un modèle global du système "Albenche" (CAZELLES, 1987) centré sur les transferts de carbone organique (éqs (1)) dont la structure est schêmatisêe à la figure 2. Basée sur le principe de conservation de masse, la formulation mathématique du modèle est constituée par un système d'équations aux dérivêes partielles qui décrivent l'évolution de chaque compaxtiment en fonction du temps et de la distance longitudinale. Cette représentation spatio-temporelle permet de coupler les processus biologiques (consommation de matière organique, par exemple) et les mécanismes physiques (transport, par exemple), démarche essentiel pour une descxiption correcte de la dynamique de l'écosystème.

$$
\begin{aligned}
& \frac{\partial C}{\partial t}=\text { transport }- \text { consommation par } \mathrm{Bi}+\text { apports } \\
& \frac{\partial C_{p s} 1}{\partial t}=\text { transport }_{i} \text { - sédimentation } i \\
& + \text { érosion }_{i}+\text { apports }_{i} \\
& \frac{\partial \mathrm{Cpb}_{1}}{\partial t}=\text { sédimentation } i \text { - érosion } i \\
& + \text { consommation }_{i} \text { par } \mathrm{Bi} \text { et } \mathrm{Ba} \\
& \text { + mortalité } j \text { de } \mathrm{Bi} \text { et } \mathrm{Ba} \\
& \frac{\partial \mathrm{Bi}}{\partial t}=\text { assimilation. consommation de } \mathrm{C} \text { et } \mathrm{Cpb}_{i} \\
& \text { - respiration - mortalité } \\
& \text { - prédation par Ba } \\
& \frac{\partial \mathrm{Ba}}{\partial \mathrm{t}}=\text { assimilation.consommation de } \mathrm{Cpb}_{\mathbf{i}} \text { et } \mathrm{Bi} \\
& \text { - respiration - mortalité } \pm \text { dérive } \\
& \text { - émergence }
\end{aligned}
$$

L'établissement des équations qui correspondent à ces différents mécanismes a étê détaillé dans un précédent travail (CAZELLES, 1987). I1 est rappelé, que seule est décrite ici la partie du modèle concernant la dynamique du carbone organique dissous et celle des microorganismes benthiques. 


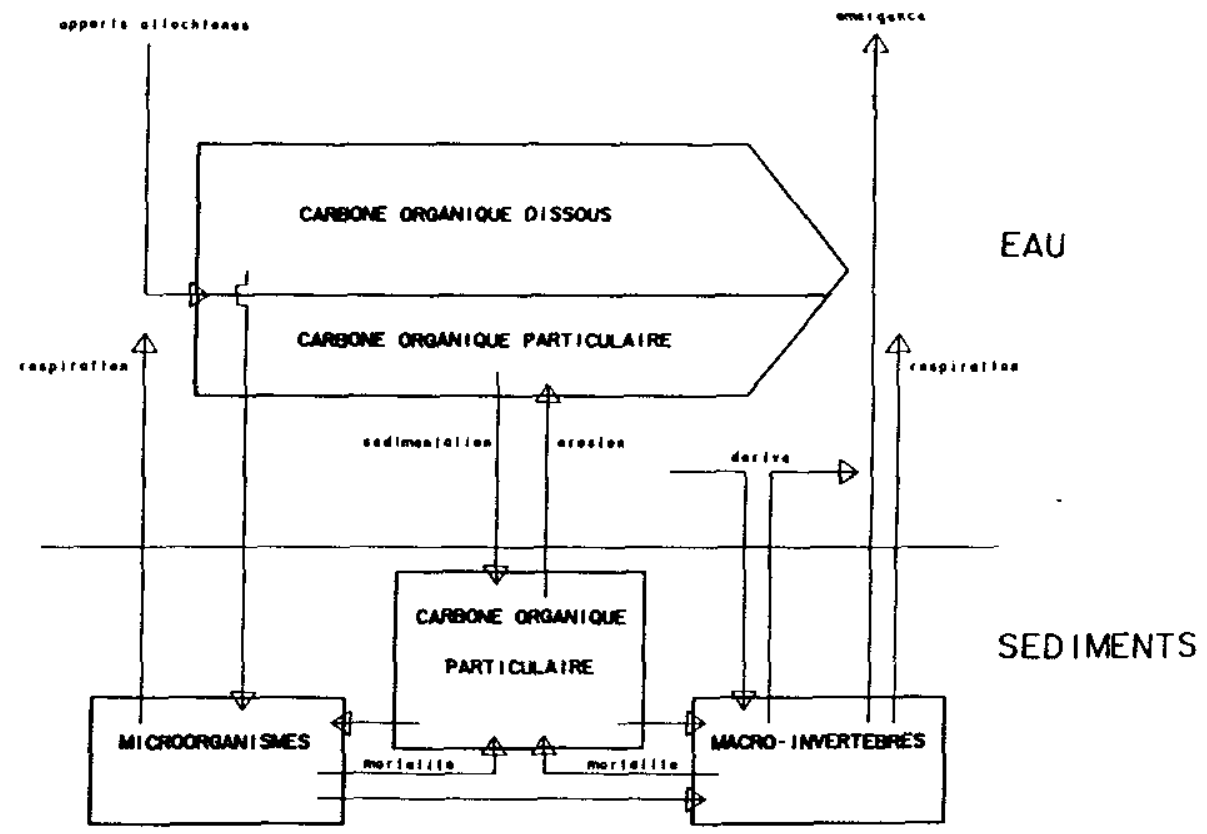

Figure 2. - Schématisation du modèle des flux de carbone organique dans l'Albenche.

Figure 2. - Schematic representation of the model simulating the carbon fluxes in the Albenche river.

4 - PRISE EN COMPTE DES MICROORGANISMES BENTHIQUES

La matière organique étant la principale source énergétique du systẻme "Albenche", les processus détritiques y représentent la majeure partie des activités biologiques du système. Un effort particulier a donc été apporté à l'écriture du sous-modêle qui décrit ces processus.

L'analyse de la bibliographie montre que les modèles écologiques d'écosystèmes aquatiques prennent rarement en compte les processus de dégradation de la matière organique malgré leur rôle prépondérant dans la structure et le fonctionnement des écosystèmes. Il est surprenant, par ailleurs, de constater que la plupart des auteurs qui modélisent la qualité des eaux et qui s'intéressent aux mécanismes de disparition de la matière organique et de consomution d'oxygène, proposent et utilisent des sous-modèles de biodégradation qui n'ont pas évolué depuis celui proposé par STREETER et PHELPS en 1925. Depuis cette date les modèles de qualité d'eau se sont certes améliorés. Cependant les meilleurs résultats sont obtenus non par une formulation plus appropriée des mécanismes de biodégradation, mais par la prise en compte d'autres processus intervenant dans les bilans globaux de la matière organique et de 1 'oxygène dissous : 
- mécanismes de mélange (dilution, convection, dispersion) ;

- autres mécanismes physiques (sédimentation, remise en suspension, biosorptions) ;

- rôle du compartiment benthique ;

- interactions avec la production primaire (photosynthèse et respixation des organismes autotrophes) ;

- processus liés aux pollutions azotées (nitrification, dénitrification).

Dans ces modèles, les phénomènes de biodégradation sont qénéralement modélisés à l'aide de cinétiques d'ordre 1 par rapport à la concentration en substrats organiques. L'utilisation de ces cinêtiques $n$ 'a aucune justification thêorique en raison de la complexité des réactions biochimiques mises en jeu. La concentration en oxygène dissous et la dégradation de la matière organique ne suivent probablement pas des cinétiques de réactions monomoléculaires à stoechiométrie constante. De plus, la décomposition de la matière organique est un processus autocatalytique, dont les principaux responsables sont les populations microbiennes. La vitesse de ces réactions doit donc ètre considérée comme une fonction de la concentration en substrat et de la quantité des microorganismes présents. Elle est également fonction d'autres facteurs environnementaux (SCHWARTZBROD, 1985) et en particulier de la température, de la nature des substrats et des populations bactériennes.

Ces mécanismes auto-catalytiques ont été pris en compte dans des modẻles classiques (MONOD, HERBERT, COMTOIS, ...) qui servent de base à la construction de la majorité des modèles complexes mis au point dans le traitement des eaux (modèles de boues activées, modèles de biofilm). Ils ont été appliqués, beaucoup plus rarement, à des modèles écologiques ou de qualité d'eau :

- GATES et al. (1969), STEHFEST (1973), RUTHERFORD et O'SULLIVAN (1974), MARTIN et LELONG (1981) ainsi que BUHR et MILLER (1983) utilisent le formalisme de MONOD :

- EDELINE et ses collaborateurs (EDELINE et BUET, 1978; EDELINE et LAMBERT, 1979) proposent un modèle de MONOD dégénéré ;

- SRINANTHAKUMAR et AMIRTHARAJAH (1983), GANTZER et al. (1988), comme KAWASHIMA et SUZUKI (1989) utilisent les concepts des modèles de biofilm;

- CLESCERI et al. (1977) ainsi que SERVAIS (1987) incorporent le formalisme de MONOD dans des modèles complexes qui tiennent compte du degré de labilité des substrats organiques.

\section{4-1 Modélisation de la dégradation de la matière organique dissoute par les microorganismes benthiques}

Les populations bactériennes fixées jouent un rôle important dans l'utilisation de la matière organique, spécialement dans les petits cours d'eau (COSTERTON et al. , 1978 ; GEESEY et al. 1978 ; SRINANTHAKUMAR et AMIRTHARAJAH, 1983 ; LOCK et al., 1984). En effet, dans ces cours d'eau le rapport surface du fond sur volume de l'écoulement est beaucoup plus important que dans les grands cours d'eau. LOCK et ses collaborateurs (LOCK, 1981; LOCK et al., 1984) ont proposé un modèle conceptuel 
pour décrire le fonctionnement, dans les petits cours d'eau, de ces communautés benthiques et épilithiques. Les principaux concepts de ce modèle sont les suivants :

- les organismes hétéxotrophes et photosynthétiques de ces communautēs se trouvent dans une matrice polysaccharidique homogène ;

- les différents substrats aiffusent dans cette matrice et sont utilisés par les différents microorganismes et leurs exoenzymes ;

- cette matrice constitue êgalement un lieu d'adsorption priviljgié pour les différents substrats particulaires.

On peut constater que ces concepts se rapprochent beaucoup de ceux introduits dans les modèles de biofilm mis au point pour simulex l'action épuratrice des biomasses fixées utilisées dans le traitement des eaux (LA MOTTA, 1976 ; WILLIAMSON et Mac CARTY, 1976 ; RITTMANN et Mac CARTY, 1978 ; TRULEAR et CHARACKLIS, 1982).

Pour modéliser les mécanismes de dégradation bactérienne dans le compartiment benthique des petits cours d'eau, on peut donc faire l'analogie avec l'activité épuratrice des biomasses fixêes. D'ailleurs, ces dernières années, plusieurs auteurs sont partis de cette analogie pour appliquer des modèles de biofilm plus ou moins simplifiês à de petits cours d'eau (SRINANTHAKUMAR et AMIRTHARAJAH, 1983 ; GANTZER et al., 1988 ; KAWASHIMA et SUZUKI, 1989).

De part leurs concepts et leur structure, ces modèles de biofilm permettent de prendre en compte la dualitê des mécanismes mis en jeu dans la dégradation et la disparition de la matière organique dans le compartiment benthique :

- mécanismes de transfert des substrats organiques, de la masse d'eau vers ce compartiment ;

- utilisation de la matièxe organique par les microorganismes et leur croissance.

Le modèle proposé pour décrire la dynamique du C.O.D. dans l'Albenche tient compte des travaux récents publiés sur les modèles de biofilm (TRULEAR et CHARACKLIS, 1982 ; KISSEL et al. 1984 ; SIEGRIST et GUJER, 1985 ; SARNER et MARKLUND, 1985 ; CHANG et RITTMANN, 1987 ; SPEITEI et al., 1987). Il inclue les mécanismes suivants :

- transfert des substrats organiques a travers l'interface eau-sédiment, diffusion de ces substrats dans le biofilm et assimilation par les populations bactériennes :

- croissance des bactéries à partir des substrats organiques, métabolisme endogène, dégénérescence, arrachement d'une partie de la biomasse et remise en suspension des produits issus de l'autolyse des bactéries dégénérées.

L'analyse du système a conduit également à l'introduction dans le modèle des hypothèses suivantes :

- dégradation de matière organique dans la veine liquide négligeable devant celle se déroulant dans le compartiment benthique; les nesures de production de $\mathrm{CO}_{z}$ effectuées in situ dans la veine liquide s'étant révélées inférieures au seuil de détection de la mesure ; 
- ècoulement turbulent assurant une bonne oxygénation aussi bien dans la masse d'eau que dans le film benthique; réactions métaboliques de biodêgradation limitêes uniquement par le carbone ;

- application du modèle aux microorganismes pris en compte par la mesure d'A.D.N. (méthode de FONTVIEILLE et FEVOTTE, 1981); cette méthode permet d'estimer la biomasse des microorganismes présents (bactëries, champignons, algues, protozoaires....) ; compte tenu de la forte charge organique que reçoit le cours d'eau, les microorganismes photosynthêtiques sont quasiment absents ; de ce fait l'A.D.N. mesuré est considêré représenter seulement les microorganismes hétérotrophes ;

- considérations des seules biodégradations imputables aux populations de microorganismes hétérotrophes dans ces conditions aérobies ;

- longueur d'échange entre le film benthique et la masse d'eạu égale au périmètre mouillé de l'écoulement (Pm).

Les équations suivantes (2) ont ainsi été obtenues :

$$
\begin{aligned}
& \frac{d C}{d t}=-\frac{K t \cdot P m \cdot}{A}(C-C f o) \\
& \frac{d B i s}{d t}=\frac{K a r r \cdot X f \cdot H f}{H} \\
& \frac{d C f o}{d t}=\frac{K t \cdot P m \cdot}{A f}(C-C f o) \\
& \frac{\partial C f}{\partial t}=D f \cdot \frac{\partial^{2} C f}{\partial z^{2}}-\left(\frac{\mu m a x}{x_{1}} \cdot \frac{C f}{K s+C f}-\frac{k_{2}}{y_{2}}+K e\right) \cdot X f \\
& \frac{\partial X f}{\partial t}=\left(\mu m a x \cdot \frac{C f}{K s+C f}-k_{2}\right) \cdot X f \\
& \frac{d B f}{d t}=\int_{0}^{H f} \frac{\partial x f}{\partial t} \cdot d z-K a r r . B f
\end{aligned}
$$

Ce modèle prend en compte l'hêtérogénéité spatiale (verticale) du compartiment benthique. Il peut permettre de suivre l'évolution de diffërents substrats à travers l'épaisseur đu biofilm, et de déterminer ainsi l'épaisseur sur laquelle s'effectue la plus grande partie de la décomposition de la matière organique.

Ces avantages sont obtenus au prix d'une complexification importante des êquations du modèle et de l'introduction dans les équations de nombreux paramètres. Pour réduire cette complexité mathématique et pour pallier les problèmes posés par la calibration et la validation d'un tel modèle (2), différentes hypothèses simplificatrices peuvent être formulées. En adoptant, par exemple, I'hypothèse de gradients de concentration réduits dans l'épaisseur des biofilms (SIEGRIST et GUJER, 1985), les équations (2) peuvent facilement être intégrées sur cette épaisseur. On obtient alors le système d'équations suivant : 


$$
\begin{aligned}
& \frac{d C}{d t}=-\frac{K t \cdot P m \cdot}{A}(C-C f) \\
& \frac{d B i s}{d t}=\frac{K a r r \cdot B f \cdot H f}{H} \\
& \frac{d C f}{d t}=\frac{K t \cdot P m \cdot}{A f} \quad(C-C f o)-\left(\frac{\mu m a x}{Y_{1}} \cdot \frac{C f}{K s+C f}-\frac{k_{2}}{Y_{2}}+K e\right) \cdot B f \\
& \frac{d B f}{d t}=\left(\mu \max \cdot \frac{C f}{K s+C f}-k_{2}-K a r r\right) \cdot B f
\end{aligned}
$$

\section{4-2 Mise au point du modèle}

Pour décrire la dynamique d'un écosystème lotique, il est essentiel de coupler le modèle biologique aux équations décrivant les phénomènes hydrophysiques qui se déroulent dans le cours d'eau. La représentation mathématique de ces phénomènes est basée sux les équations de bilan des eaux de surface (FABRE et RAMANY BALA, 1980; CAUSSADE et al., 1982). Deux sous-modèles hydrophysiques ont été élaborés à partir de ces équations (CAZELLES, 1987) :

- un sous-modèle hydrodynamique dérivé du modèle de saint-Venant, dont on a gardé l'équation de conservation de masse et remplacé l'équation dynamique pax une équation empirique :

- un sous-modèle de transport, constitué par l'équation classique de convection-dispersion appliquée à un écoulement monodimensionnel, nonuniforme et non-stationnaire.

Cette partie hydrophysique a été mise au point à l'aide de campagnes de traçage réalisées avec un colorant, la rhodamine B (CAZELLES, 1987 ; CAZELLES et FOITVIEILLE, 1989). La figure 3 illustre certains des résultats donnés par ces sous-modèles.

Le modèle complet est constituê par les équations :

- (4) pour le sous-modèle hydrodynamique ;

- (5) pour le sous-modèle de transport dans lequel a été ajouté un terme traduisant les transferts à l'interface eau-film; les équations (6) expriment le coefficient de dispersion en fonction de certaines caractéristiques de l'écoulement (LIU, 1977) :

- (7) pour le sous-modèle écologique ;

- (8) pour exprimer l'influence de la température sur les paramètres du métabolisme des microorganismes.

$$
\begin{aligned}
& \frac{\partial A}{\partial t}+\frac{\partial Q}{\partial x}=q \\
& A=c a \cdot Q \text { ea } \\
& \frac{\partial A \cdot C}{\partial t}+\frac{\partial Q \cdot C}{\partial x}=\frac{\partial}{\partial x}\left(A \cdot D_{L} \cdot \frac{\partial C}{\partial x}\right)+C q \cdot q-P m \cdot K t \cdot(C-C f)
\end{aligned}
$$



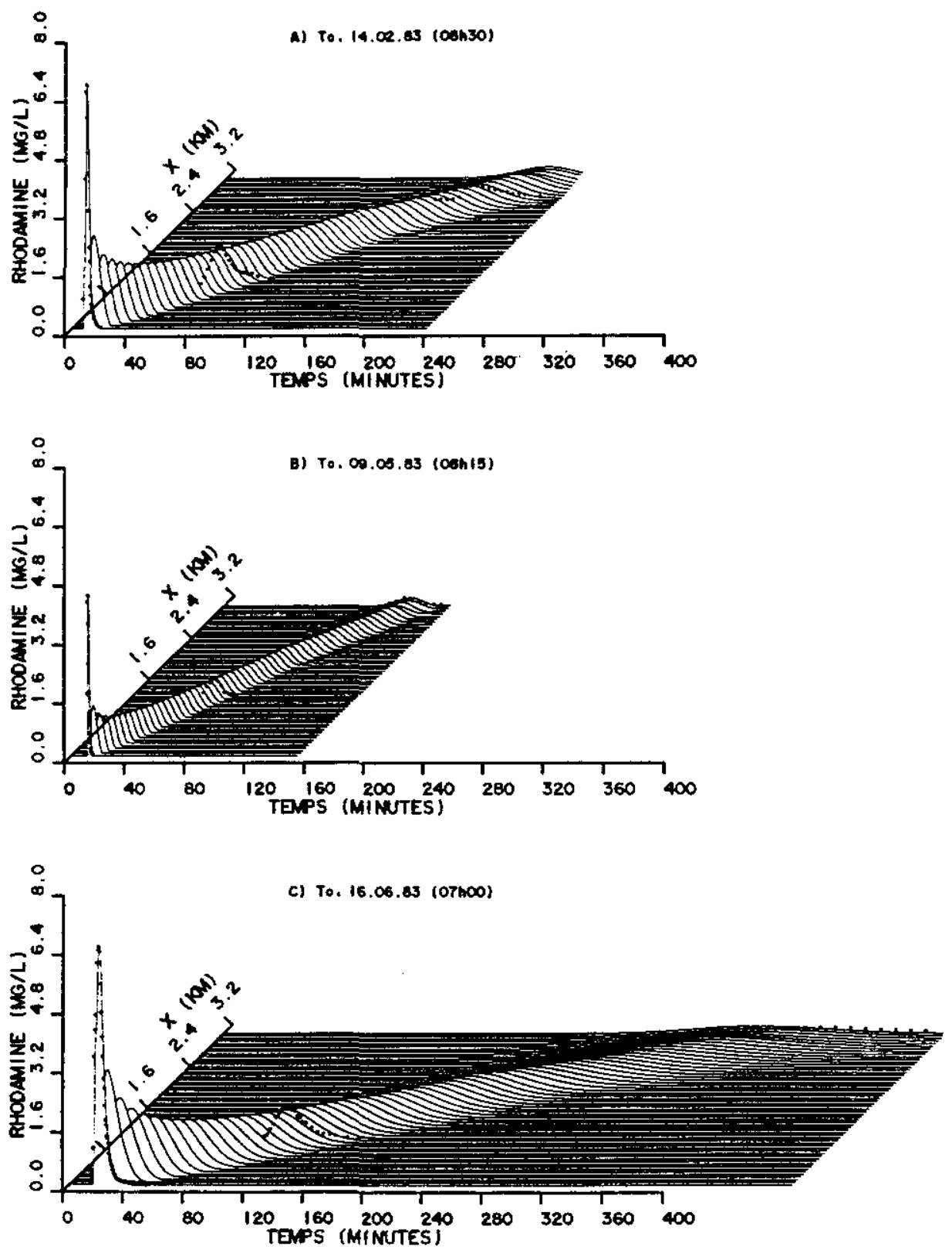

Figure 3. - Simulation d'expériences de traçage dans l'Albenche. ( $\mathrm{a}: \mathrm{Q}=165 \mathrm{l} / \mathrm{s}, \mathrm{b}: \mathrm{Q}=3951 / \mathrm{s}, \mathrm{c}: \mathrm{Q}=77 \mathrm{1} / \mathrm{s}$, * : valeurs experimentales).

Figure 3. - Simulation of dye tracer experiments in the Albenche river. $(a: Q=165 \mathrm{l} / \mathrm{s}, b=Q=395 \mathrm{l} / \mathrm{s}, c=Q=77 \mathrm{l} / \mathrm{s}$, * : experimental values). 


$$
\begin{aligned}
& \left.\begin{array}{rl}
D_{L} & =\frac{B D L \cdot U^{2} \cdot B^{3}}{U p^{*} \cdot A} \\
B \dot{D}_{L} & =\alpha i \cdot\left(\frac{U}{U p^{*}}\right) B i
\end{array}\right] \\
& \left.\frac{\partial A f \cdot C f}{\partial t}=P m \cdot K t \cdot(C-C f)-\left(\frac{\mu \max }{Y_{1}} \cdot \frac{C f}{K s+C f}-\frac{k_{2}}{Y_{2}}+K e\right) \cdot B f \cdot P m\right] \\
& \frac{\partial P m \cdot B f}{\partial t}=\left(\mu \max \cdot \frac{C f}{K s+C f}-k_{2}-K a r r\right) \cdot B f \cdot P m \\
& \kappa \mathrm{p}, \mathrm{T}=\kappa \mathrm{p}, 20^{\circ} \cdot \theta^{\left(\mathrm{T}-20^{\circ}\right)}
\end{aligned}
$$

La simulation de la dynamique du C.O.D. et des microorganismes benthiques de l'Albenche à l'aide de ce modèle, est réalisée sur la base des hypothèses suivantes :

- les valeurs des paramètres des sous-modèles physiques sont celles obtenues lors du calage de ces sous-modèles à l'aide des campagnes de traçage:

- le cycle de 24 heures des flux horaires mesurés à l'entrểe du tronçon étudié se reproduit pendant toute la durée de la simulation;

- les concentrations de l'A.D.N. microbien benthique sont en équilibre avec la charge organique du cours d'eau en $1^{\prime a b s e n c e ~ d e ~ f o r t e s ~ v a r i a-~}$ tions de température et de dêbit; ces concentrations sont donc considérées comene relativement constantes autour d'une date donnée ;

- la densité cellulaire des microorganismes du biofilm est constante et leur croissance se traduit par une augmentation de la hauteux du film ; Ie calcul de la section du biofilm par les équations (9) traduit ces hypothèses.

$$
\left.\begin{array}{l}
\text { Af }=\text { Pm.Hf } \\
H f=H_{\text {Af }} \cdot \text { Bf }
\end{array}\right]
$$

Ires êquations du modèle sont résolues par des méthodes numériques aux différences finies, utilisant des schêmas de discrêtisation implicite (CAZELLES, 1987). Les mesures effectuées le 15 août 1983 ont été choisies, arbitrairement, pour ajuster les parametres de la partie biologique du modèle. Une première estimation de l'ordre de grandeur des valeurs des paramêtres de la partie biologique du modèle, a été faite en compulsant les travaux d'auteurs ayant travaillé sur les modèles de biofilm et sur les cinétiques de croissance de populations bactériennes fixẽes mesurees in situ (KAPLAN et BOTT, 1983 ; SRINANTHAKUMAR et AMIRTHARAJAH, 1983 ; KAPLAN et BOTT, 1985) ou in vitro (OHGAKI et al., i 978 ).

Plusieurs ajustements successifs ont conduit au lot de paramètres du tableau 2, a partir duquel ont été obtenues les évolutions spatiales et temporelles présentées sur la figure 4. Trois remarques peuvent être formulées à la suite de la comparaison des valeurs retenues pour ces paramètres (tableau 2) à celles utilisées dans les modèles de biofilm décrits dans la bibliographie: 
- le taux de transfert (Kt) est faible ; ce résultat peut être la conséquence, d'une part, de l'activité des macroinvertébrés qui, par leur action locomotrice, détruisent la couche laminaire à l'interface eaufilm (Mac CALL et FISCHER, 1980 ; FRY, 1982), d'autre part, de l'épaisseur importante des biofilms étudiés poux lesquels la couche laminaire a un rôle de barrière physique déjà réduit (SIEGRIST et GUJER, 1985) ;

- le taux de croissance maximal ( $\mu$ max) est faible; cette valeur est probablement liée au fait que nous avons englobé dans le terme microorganismes, les bactéries, les champignons et les protozoaires présents ; il n'est donc pas étonnant que le taux de croissance maximal moyen de ces populations soit inférieur au taux de croissance maximal des populations bactëriennes prises en compte dans les autres modèles ;

- la valeur du taux de dégénérescence est forte; ce terme qui englobe, dans notre cas, la mortalité et la prédation, laisse supposer que la prédation par les macroinvertébrés, et/ou les phénomènes d'inhibitions métaboliques (excès de substrats organiques, dêficit d'oxygène dissous, ...) sont importants dans ce cours d'eau.

Tableau 2.- Valeurs des paramètres des modèles utilisées lors des simulations de la dynamique du C.O.D. et des microorganismes benthiques.

Table 2. - Parameter's values used by the models during the simulations of Dissolved Organique Carbon and benthic microorganisms.

Paramètres $\begin{gathered}\text { Valeurs } \\ \text { nominales }\end{gathered}$ Signification

Sous-modèles physiques

$\begin{array}{ll}\mathrm{cq} & 1.289 \\ \mathrm{eq} & 0.955 \\ \mathrm{ca}_{1} & 1.100 \\ \mathrm{ea} & 0.300 \\ \mathrm{ca}_{2} & 1.400 \\ \mathrm{ea} & 0.250 \\ \alpha_{1} & 0.153 \\ \beta_{1} & -3.607 \\ \alpha_{2} & 0.492 \\ \beta_{2} & -3.768\end{array}$

- Paramètres du calcul de la relation entre le débit et le débit d'apport - Paramètres de la relation entre $Q$ et A pour le premier et le deuxième tronçon

- Paramètres de la relation permettant le calcul du coefficient de dispersion longitudinale (DL) pour le premier et le deuxième tronçon

Sous-modèles écologiques

\begin{tabular}{|c|c|c|}
\hline $\mathrm{Kt}$ & $7.510^{-5}$ & - Vitesse de transfert à $l^{\prime}$ interface $\left(\mathrm{m} . \mathrm{s}^{-1}\right)$ \\
\hline$\mu \max$ & $5.510^{-5}$ & - Taux de croissance maximal $\left(s^{-1}\right)$ \\
\hline Ks & 300 & - Constante de demi-saturation $\left(\mathrm{g} \cdot \mathrm{m}^{-3}\right)$ \\
\hline$Y_{1}$ & 0.04 & - Coefficient de conversion \\
\hline$k_{2}$ & $1.110^{-5}$ & - Taux de dégếnérescence $\left(\mathrm{s}^{-1}\right)$ \\
\hline$Y_{2}$ & 20 & - Coefficient de remétabolisation \\
\hline ke & $2.510^{-5}$ & - Taux de métabolisme endogène $\left(s^{-1}\right)$ \\
\hline Karx & 5. $10^{-6}$ & - Taux d'arrachement $\left(s^{-1}\right)$ \\
\hline $\mathrm{H}_{\mathrm{A}} \mathrm{f}$ & $3.510^{-4}$ & $\begin{array}{l}\text { - Coefficient permettant le calcul de } \\
\text { Hf }\left(\mathrm{m}^{3} \cdot \mathrm{g}^{-1}\right)\end{array}$ \\
\hline$\theta$ & 1.024 & $\begin{array}{l}\text { - Paramètre exprimant l'influence de la } \\
\text { température }\end{array}$ \\
\hline
\end{tabular}




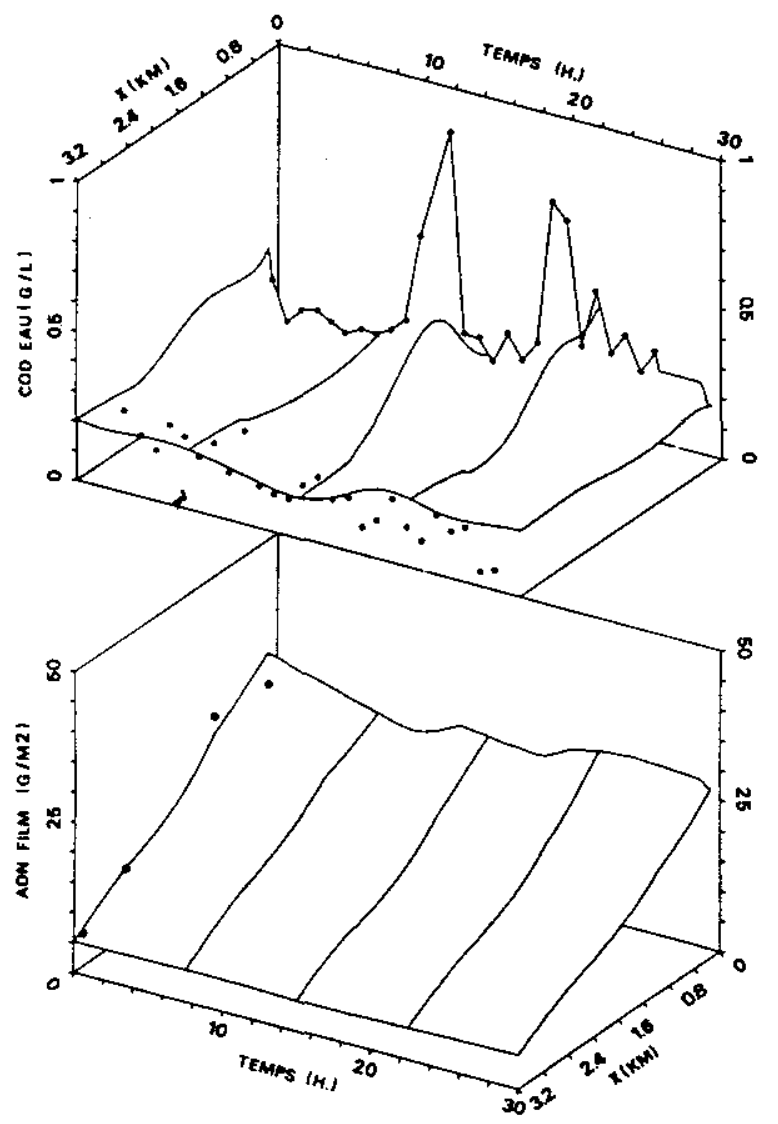

Figure 4. - Simulation de la dynamique du carbone organique dissous et de 1'A.D.N. microbien benthique dans l'Albenche $($ To $=15.08 .83 \mathrm{a} 22 \mathrm{~h})$. Les valeurs expérimentales $(*)$ se trouvent dans les plans $x=0.150 \mathrm{~km}$ (arrière plan de la figure, station amont) et $x=3.200 \mathrm{~km}$ (premier plan de la $\mathrm{fi}-$ gure, station aval). Les caractéristiques de cette période sont les suivantes :

- débit : 20 à $30 \mathrm{l} . \mathrm{s}^{-1}$ pour la station amont, 32 à $451 . \mathrm{s}^{-1}$ pour la station aval

- température : 14 à $17^{\circ} \mathrm{C}$

- concentration des apports (Cq) : 120 à $150 \mathrm{mg} \cdot \mathrm{l}^{-1}$.

Figure 4. - Simulation of the dissolved organic matter dynamics in the Albenche river (To $=15.08 .83$ at $22 \mathrm{~h}$ ). The experimental values (*) are plotted on the background plane of the picture $(x=0.150 \mathrm{~km}$. upstream station) and on the foreground plane of the picture $(x=3.200 \mathrm{~km}$, downstream station). The main characteristics of the period under consideration are :

- discharge : 20 d 30 Z. $\mathrm{s}^{-1}$ for upstream station. $32 \grave{a} 45$ l. $s^{-1}$ for downstream station

- temperature : $14 \grave{a} 17^{\circ} \mathrm{C}$

- concentration of the allochtonous diffuse organic carbon inputs $(\mathrm{Cq}): 120$ a $150 \mathrm{mg} \cdot \mathrm{l}^{-1}$. 
Une vếrification du modèle (CAzELLES, 1987) a êté effectuée par un essai de comportement, en faisant varier fortement chacun des paramètres du modèle. Elle a permis de constater que la structure du modèle adoptée décrit correctement les hypothèses envisagêes et ne pose pas de problème d'instabilité structurelle.

Une validation a ensuite êté conduite en simulant la dynamique du C.O.D. et de I'A.D.N. microbien benthique à des dates différentes de celles utilisées pour la calibration đu modèle 104.10 .83 et période du $07.98 \mathrm{au} 15.08 .83)$, avec le même lot de paramètres (tableau 2).

Les résultats obtenus sont présentếs sur les figures 5 et 6 . Ils sont tout à fait satisfaisants en regard du faible nombre (A.D.N.) et de la grande variabilité (C.O.D.) des données expérimentales à partir desquelles l'ajustement de la partie biologique du modèle a été réalisé.

5 - APPLICATIONS DU MODĖLE

La modélisation, en gënéral, $n$ 'est pas une fin en soi, mais un outil cognitif puissant qui permet d'établir des bilans, de tester le rôle de certains phênomènes et de rechercher les effets de certains facteurs environnementaux sur la dynamique des systèmes étudiês. Il nous est donc apparu intéressant d'utiliser le modèle sous sa forme actuelle, malgré ses imperfections, pour essayer d'accroitre la connaissance du fonctionnement du système "Albenche". Il est important, cependant, de garder en mémoire lors de toute application, que les résultats obtenus ne sont que le reflet des valeurs et des hypothèses introduites dans le modèle.

Le modèle a permis, tout d'abord, de quantifier l'importance des mécanismes biochimiques et biologiques liès au film benthique, pour la réduction de la charge organique de l'eau. Cette estimation s'effectue par comparaison des simulations réalisées par le modèle total avec celles réalisées par un modèle constitué uniquement des sous-modèles hydrophysiques (figures 7 et 8). Il apparait ainsi que la disparition de la charge organique est favorisée par une faible convection, une forte température et par 1'importance de la biomasse présente.

Une seconde application a été réalisée en utilisant la simulation effectuée entre le 7 et le 16 août 1983. Elle consiste à calculer, pour cette période, les valeurs des différents flux moyens de carbone organique (figure 9) ainsi que les bilans de C.O.D. de l'ensemble du tronçon étudié (figure 10), Les flux de respiration sont estimés à partir de la relation suivante :

$$
\frac{\partial \text { Cresp }}{\partial t}=\operatorname{Kresp} \cdot\left(\mu \max \cdot \frac{\mathrm{Cf}}{\mathrm{Ks}+\mathrm{Cf}}+\mathrm{k}_{2}+\mathrm{Ke}\right) \cdot \text { Bf.Pm }
$$

La valeur du paramètre Kresp est ajustée à l'aide de valeurs de production de $\mathrm{CO}_{2}$ mesurées dans la station 1 durant cette période, en admettant que la respiration des macroinvertébrés est négligeable devant celle des microorganismes. 


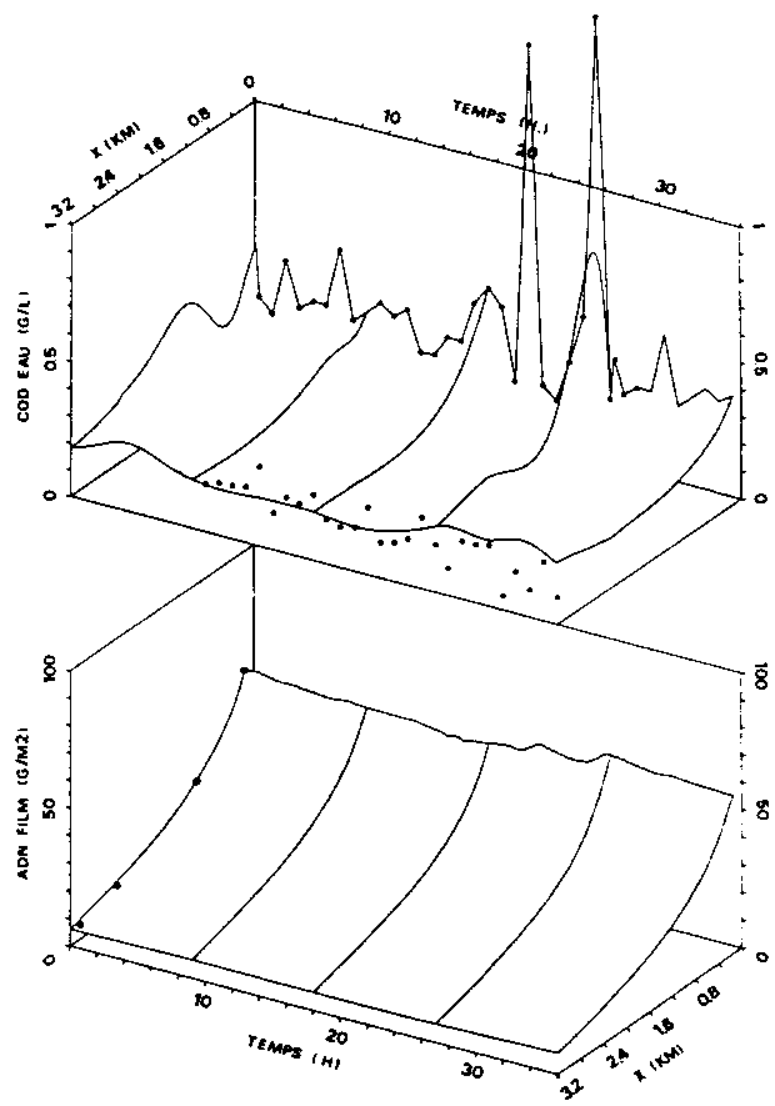

Figure 5. - Simulation de la dynamique du carbone organique dissous et de I'A.D.N. microbien benthique dans l'Albenche (To $=04.10 .83$ a $18 \mathrm{~h}$ ). Les valeurs expérimentales $(*)$ se trouvent dans les plans $x=0.150 \mathrm{~km}$ (arrière plan de la figure, station amont) et $x=3.200 \mathrm{~km}$ (premier plan de la $\mathrm{fi}$ gure, station aval). Les caractéristiques de cette période sont les suivantes:

- débit : 30 à $381 . \mathrm{s}^{-1}$ pour la station amont, 45 à $571 . \mathrm{s}^{-1}$ pour la station aval

- température : 9.5 à $11.5^{\circ} \mathrm{C}$

- concentration des apports (Cq) : 250 à $340 \mathrm{mg} \cdot \mathrm{l}^{-1}$.

Figure 5. - Simulation of the dissolved organic matter dynamics in the Albenche river (To $=04.10 .83$ at $18 \mathrm{~h})$. The experimental values (*) are plotted on the background plane of the picture $(x=0.150 \mathrm{~km}$. upstream station) and on the foreground plane of the picture $(x=3.200 \mathrm{~km}$. downstream station). The main characteristics of the period under consideration are :

- discharge : 30 à 38 Z.s $\mathrm{s}^{-1}$ for upstream station, 45 à 57 Z. $s^{-1}$ for downstream station

- temperature : 9.5 à $11.5^{\circ} \mathrm{C}$

- concentration of the allochtonous diffuse organic carbon inputs (Cq) : 250 à $340 \mathrm{mg} \cdot \mathrm{l}^{-1}$. 


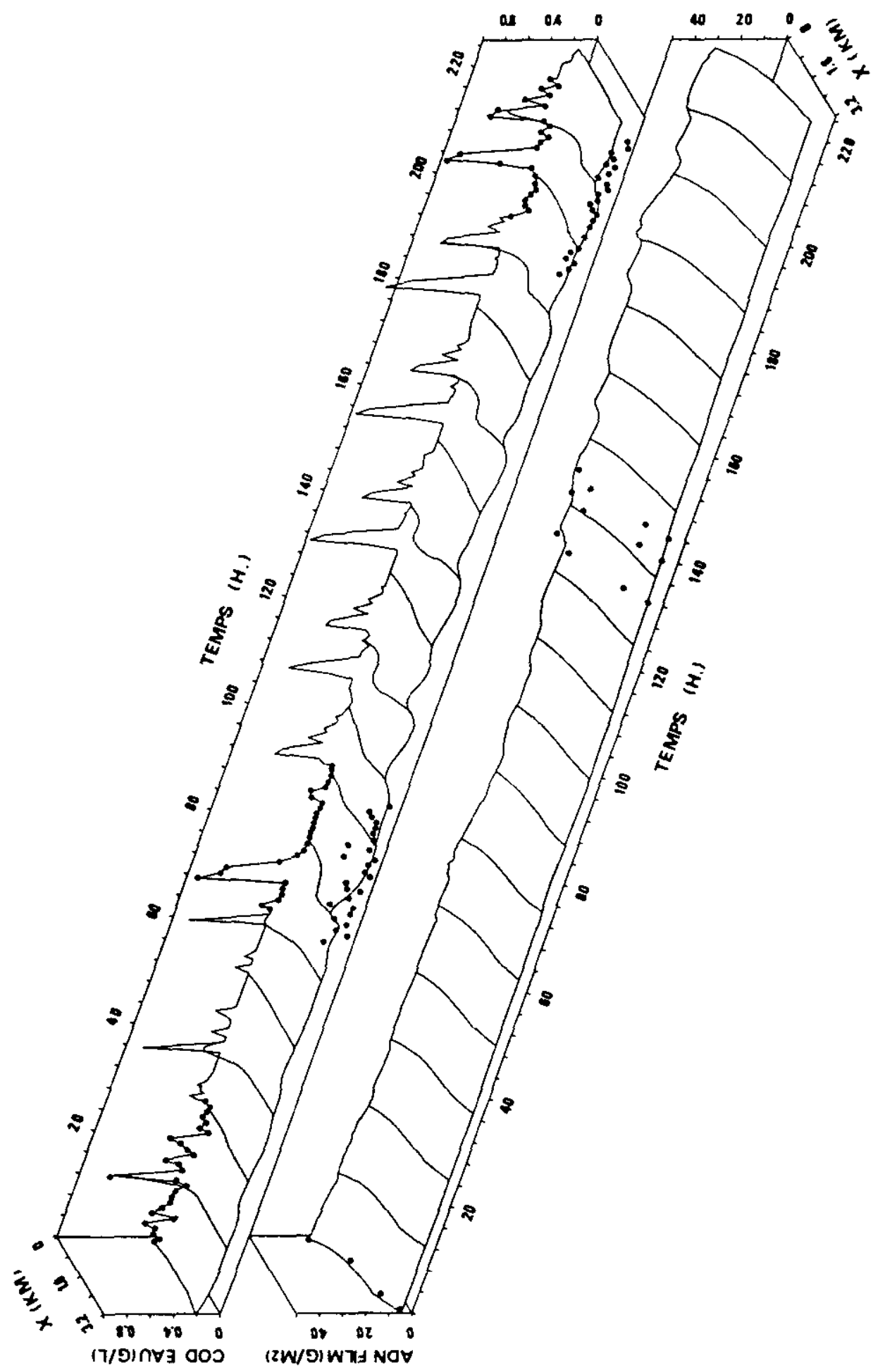


Figure 6. - Simulation de la dynamique du carbone organique dissous et de I'A.D.N. microbien benthique dans 1 'Albenche (To $=07.08 .83 \mathrm{a} 22 \mathrm{~h}$ ). Les valeurs expérimentales (*) se trouvent dans les plans $x=0.150 \mathrm{~km}$ (arrière plan de la figure, station amont) et $x=3.200 \mathrm{~km}$ (premiex plan de la $\mathrm{fi}-$ gure, station aval). Les caracteristiques de cette période sont les suivantes :

- débit : 20 a $951 . \mathrm{s}^{-1}$ pour la station amont, 40

a $1451 . \mathrm{s}^{-1}$ pour la station aval

- température : 12 a $17^{\circ} \mathrm{C}$

- concentration des apports (Cq) : 120 a $150 \mathrm{mg} \cdot 1^{-1}$.
Figure 6. - Simulation of the dissolved organic matter dynamics in the Albenche river (To = 07.08 .83 at $22 \mathrm{~h}$ ). The experimental values (*) are plotted on the background plane of the picture $(x=0.150 \mathrm{~km}$. upstream station) and on the foreground plane of the picture $(x=3.200 \mathrm{~km}$. downstream station). The main characteristics of the period under consideration are :

- discharge : 20 à $95 \quad 2 . \mathrm{s}^{-1}$ for upstream station. 40 \& $1452 . \mathrm{s}^{-1}$ for downstream station

- temperature : $12 d 17^{\circ} \mathrm{C}$

- concentration of the allochtonous diffuse organic carbon inputs $(\mathrm{Cq}): 120$ i $150 \mathrm{mg} \cdot \mathrm{l}^{-1}$. 


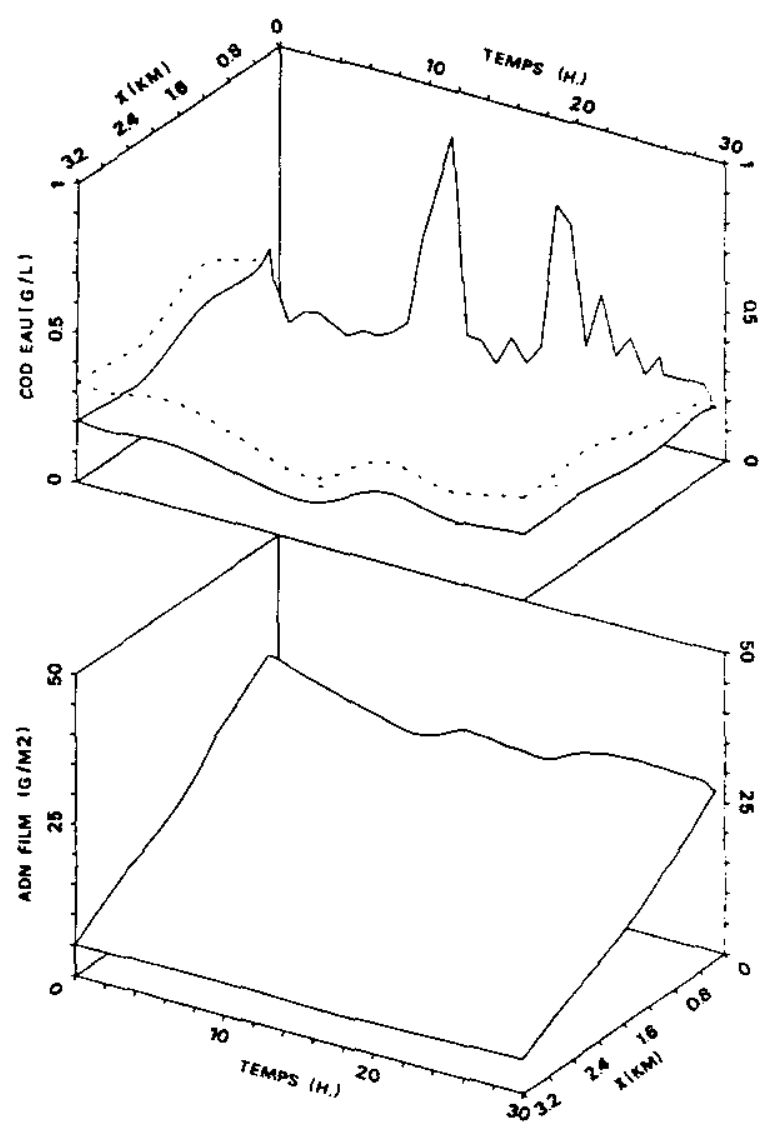

Figure 7. - Rôle du film benthique dans l'êlimination de la charge organique de $l$ 'eau le 15.08.83. L'activité des microorganismes benthiques entraîne une diminution de la charge organique de $2.5 \mathrm{\&}$ au point $200 \mathrm{~m}$, de 26.5 au point $1000 \mathrm{~m}$ et de 37 \& au point $3200 \mathrm{~m}$. Les caractéristiques de cette période sont les suivantes :

- débit : 20 à $301 . \mathrm{s}^{-1}$ pour la station amont, 32 a $451 . \mathrm{s}^{-1}$ pour la station aval

- température : 14 à $17^{\circ} \mathrm{C}$

- concentration des apports (Cq) : 120 à $150 \mathrm{mg} \cdot \mathrm{1}^{-3}$

(- modèle total ; -...-.-. sous-modèles hydrophysiques).

Figure 7. - Influence of the benthic biofilm on the removal of the organic load $(15.08 .83)$. The benthic microorganism activity is accountable for the removal of $2.5 \%$ of the organic load at the point $200 \mathrm{~m}$, of $26.5 \%$ at the point $1000 \mathrm{~m}$ and of $37 \%$ at the point $3200 \mathrm{~m}$. The main characteristics of the period under consideration are :

- discharge : 20 à 30 l. $\mathrm{s}^{-1}$ for upstream station. 32 à 45 2. $\mathrm{s}^{-1}$ for downstream station

- temperature : $14 \grave{\mathrm{a}} 17^{\circ} \mathrm{C}$ concentration of the allochtonous diffuse organic carbon inputs (Cq) : 120 à $150 \mathrm{mg} \cdot \mathrm{t}^{-1}$

(- global model; 


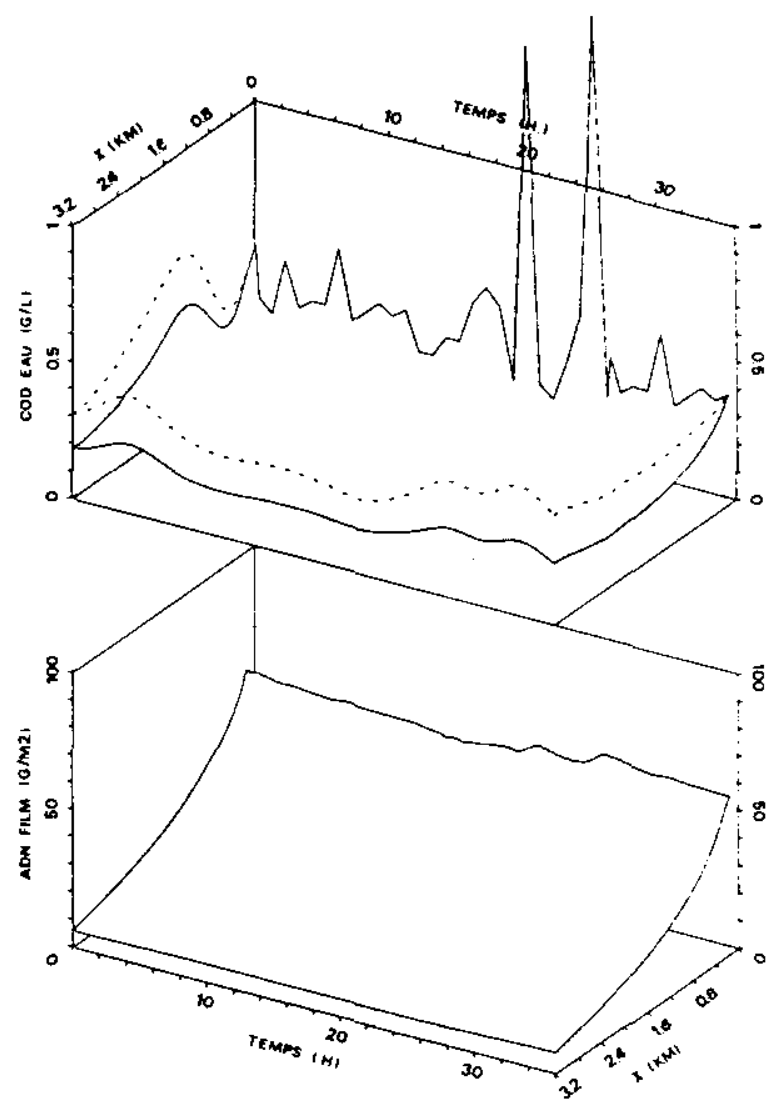

Figure 8. - Rôle du film benthique dans l'élimination de la charge organique de l'eau le 04.10.83. L'activité des microorganismes benthiques entraine une diminution de la charge organique de 3.5 au point $200 \mathrm{~m}$, de 31.5 au point $1000 \mathrm{~m}$ et de 41.5 au point $3200 \mathrm{~m}$. Les caractéristiques de cette période sont les suivantes :

- débit : 30 a $381 . \mathrm{s}^{-1}$ poux la station amont, 45 à $57 \mathrm{l} . \mathrm{s}^{-1}$ pour la station aval

- température : 9.5 a $11.5^{\circ} \mathrm{C}$

- concentration des apports (Cq) = 250 à $340 \mathrm{mg} \cdot \mathrm{I}^{-1}$

(- modèle total ; - ------- sous-modèles hydrophysiques).

Figure 8. - Influence of the benthic biofilm on the removal of the organic load (04.10.83). The benthic microorganism activity is accountable for the removal of $3.5 \%$ of the organic load at the point $200 \mathrm{~m}$, of $31.5 \%$ at the point $1000 \mathrm{~m}$ and of $41.5 \%$ at the point $3200 \mathrm{~m}$. The main characteristics of the period under consideration are :

- discharge : $30 \grave{a} 38 \mathrm{l} \cdot \mathrm{s}^{-1}$ for upstream station. $45 \grave{a}$ 57 l. $\mathrm{s}^{-1}$ for downstream station

- temperature : 9.5 a $11.5^{\circ} \mathrm{C}$

- concentration of the allochtonous diffuse organic carbon inputs $(\mathrm{Cq})$ : 250 a $340 \mathrm{mg} \cdot \mathrm{l}^{-1}$

(- alobal model ; --.--- hydrophysic sub-models). 

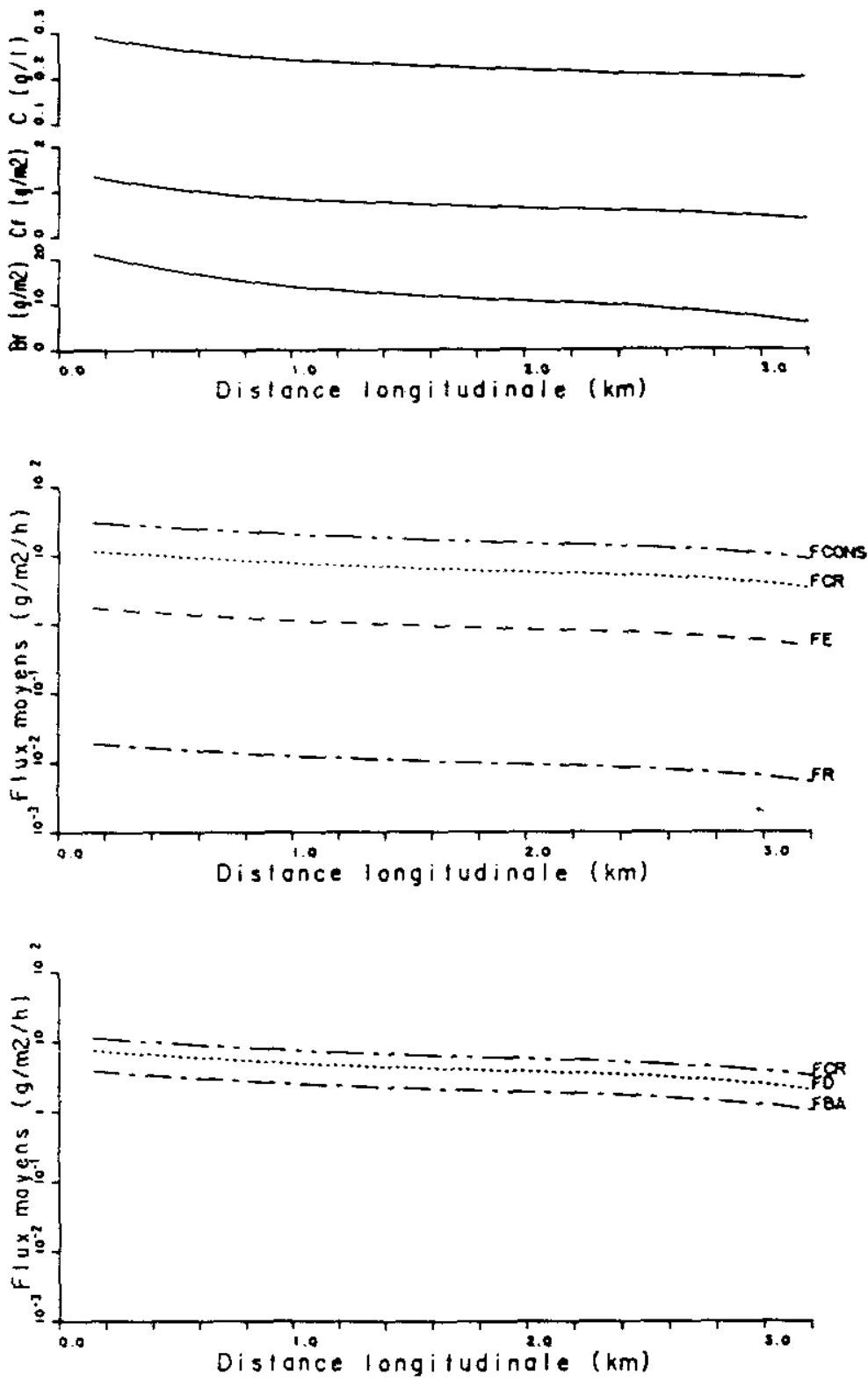

Figure 9. - Variation longitudinale des flux moyens de carbone organique entre le 7 et le 16.08.83 (FKT=transfert dans le film, FR=respiration, FCONS=consomation par les microorganismes, FCR=croissance, FE=métabolisme endogène).

Figure 9. - Longitudinal variation of the average organic carbon fluxes between the 7 th and the 16th of august 1983 (FKT= transfer into the film, FR=respiration. FCONS=microorganisms consumption. FCR=growth, $F E=e n d o g e n o u s$ metabolism). 
B'
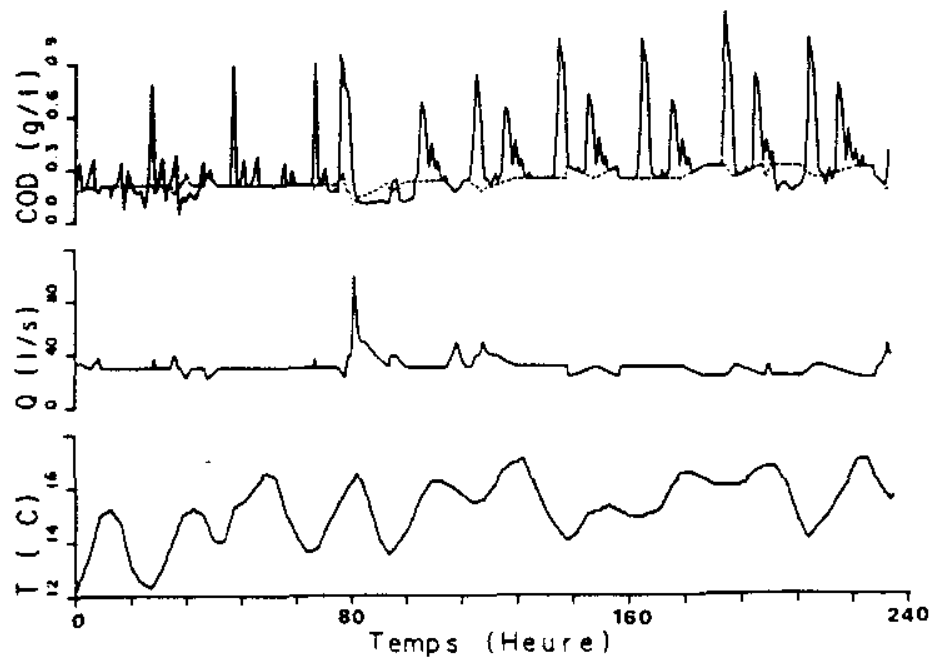

A

$\mathrm{S}: 8599.4 \mathrm{~m} 2$

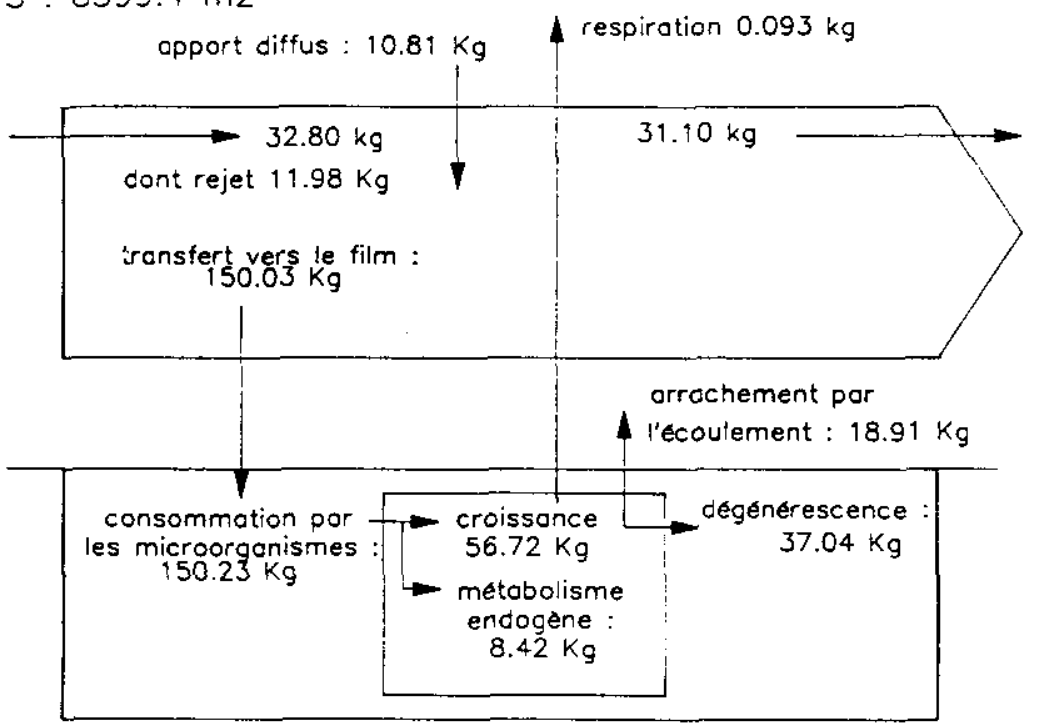

Figure 10. - Bilans horaires moyens des flux de carbone organique dissous au niveau des stations 1 et 4 de 1 'Albenche entre le 7 et le 16.08.83.

Figure 10. - Balance of the mean hour fluxes of the dissolved organic carbon at station 1 and 4 . between the 7 th and the 16 th of august 1983. 
Les calculs des bilans montrent (figures 9 et 10) que les valeurs des flux de carbone respiré, qui représentent 1'auto-épuration au sens strict du terme (FONTVIEILLE, 1978), sont très faibles. Ces flux représentent, en effet, environ 1 \& des flux de carbone consommés par les microorganismes. Cette faible valeur est d'ailleurs, en accord avec les résultats de HICKEY (1988) qui a mesuré la respiration et la consommation $a^{\prime}$ 'oxygène de la couverture biologique d'un petit cours d'eau.

Dans ce type de cours d'eau, l'épuration de la matière organique dissoute par les microorganismes procéderait, donc essentiellement par la formation et l'entretien de biomasse.

Une autre application a été effectuée par l'introduction de différentes catégories de populations microbiennes dans le modèle pour rendre compte des successions écologiques qui se pxoduisent lors de l'évolution, dans l'espace et dans le temps, de l'êtat.trophique d'un système. Une démarche analogue a ẽté suivie :

- par MARTIN et LELONG (1981) pour différentes populations phytoplanctoniques et bactériennes dans un écosystème marin expérimental ;

- par KISSEL et al. (1984) et par WANNER et GUJER (1985) pour les microorganismes autotrophes et hétérotrophes qui se succèdent dans un biofilm en fonction de son degré d'aérobiose.

Dans notre cas, deux populations microbiennes, chacune caractérisếe par des exigences nutritives particulières, ont étê considérées :

- une population "autochtone", dont le taux de croissance maximal ( maxa) est faible, dont 1 'affinité pour les substrats organiques est élevée (Ks faible), et dont les dépenses du mëtabolisme endogène sont peu importantes (Ke faible) ;

- une population "saprophile" plus adaptëe aux fortes concentrations de C.O.D. liêes aux rejets de la porcherie, dont le taux de croissance est important ( $\mu$ max élevé) et dont les dépenses a'entretien métabolique sont êgalement élevées (Ke êlevé).

L'équation du sous-modèle écologique s'écrit alors :

$$
\begin{aligned}
& \frac{\partial A f \cdot C f}{\partial t}=P m \cdot K t \cdot(C-C f)\left.-\frac{\mu m a x_{1}}{Y_{1}} \cdot \frac{C f}{K_{1}+C f}-\frac{k_{2}}{Y_{2}}+K e_{1}\right) \cdot B f_{1} \cdot P m \\
&\left.-\frac{\mu m_{2}}{Y_{1}} \cdot \frac{C f}{{K s_{2}}_{2}+C f}-\frac{k_{2}}{Y_{2}}+K e_{2}\right) \cdot B f_{2} \cdot P m \\
& \frac{\partial P m \cdot B f_{1}}{\partial t}=\left(\max _{1} \cdot \frac{C f}{K s_{1}+C f}-k_{2}-K a r r\right) \cdot B f_{1} \cdot P m \\
& \frac{\partial P m \cdot B f_{2}}{\partial t}=\left(\mu \max _{2} \cdot \frac{C f}{K_{s_{2}}+C f}-k_{2}-K a r r\right) \cdot B f_{2} \cdot P m
\end{aligned}
$$

Le tableau 3 donne les valeurs des paramètres utilisés lors de la simulation. L'évolution particulière de chacune des deux populations est représentée sur la figure 11 . 
Tableau 3. - valeurs des paramêtres des modèles utilisées lorsque deux populations de microorganismes sont considérées.

Table 3. - Parameters values used by the models when two microorganism populations are simulated:

.. a allochtone population with low values for umax. Ks and $\mathrm{Ke}$;

- a saprophile population with a hight affinity for organic wastes and high values for $\mu$ max and $K s$ but with a low value for $\mathrm{Ke}$.

\begin{tabular}{|c|c|c|}
\hline \multirow{2}{*}{ Paramètres } & \multicolumn{2}{|c|}{ Valeurs } \\
\hline & Population "saprophile" & Population "allochtone" \\
\hline $\mathrm{cq}$ & 1.289 & 1.289 \\
\hline eq & 0.955 & 0.955 \\
\hline $\mathrm{ca}_{1}$ & 1.100 & 1.100 \\
\hline$e_{1}$ & 0.300 & 0.300 \\
\hline $\mathrm{ca}_{2}$ & 1.400 & 1.400 \\
\hline$e a_{2}$ & 0.250 & 0.250 \\
\hline$\alpha_{1}$ & 0.153 & 0.153 \\
\hline$B_{2}$ & -3.607 & -3.607 \\
\hline$\alpha_{2}$ & 0.492 & 0.492 \\
\hline$\beta_{2}$ & -3.768 & -3.768 \\
\hline Kt & $7.510^{-5}$ & $7.510^{-5}$ \\
\hline umax & $7.510^{-5}$ & 3. $10^{-5}$ \\
\hline $\mathrm{Ks}$ & 600 & 150 \\
\hline $\mathrm{Y}_{1}$ & 0.04 & 0.04 \\
\hline$k_{2}$ & $1.110^{-5}$ & $1.110^{-5}$ \\
\hline$Y_{2}$ & 20 & 20 \\
\hline $\mathrm{Ke}$ & 3. $10^{-5}$ & 5. $10^{-6}$ \\
\hline Karr & 5. $10^{-6}$ & 5. $10^{-6}$ \\
\hline HAf & 4. $10^{-4}$ & 3. $10^{-4}$ \\
\hline$\theta$ & 1.024 & 1.024 \\
\hline
\end{tabular}

\section{6 - CONCLUSIONS}

En nous appuyant sur une synthèse des données et des connaissances acquises depuis 1981 sur 1'Albenche, petit cours d'eau pollué par une forte charge organique, nous avons construit un modêle mathématique de ce système écologique. Le but de ce mođèle est d'élaborer un outil cognitif qui intègre toute l'information disponible, dans l'état actuel de nos connaissances, sur le fonctionnement du système "Albenche", afin de déterminer et d'interpréter le rôle des principaux mécanismes participant à l'auto-épuration dans les cours d'eau.

Compte tenu des données expérimentales dont nous disposions, nous avons restreint, đans un premier temps, l'application du modèle global élaboré (éqs (1)) et simulé uniquement la dynamique du carbone organique dissous et des microorganismes benthiques. 


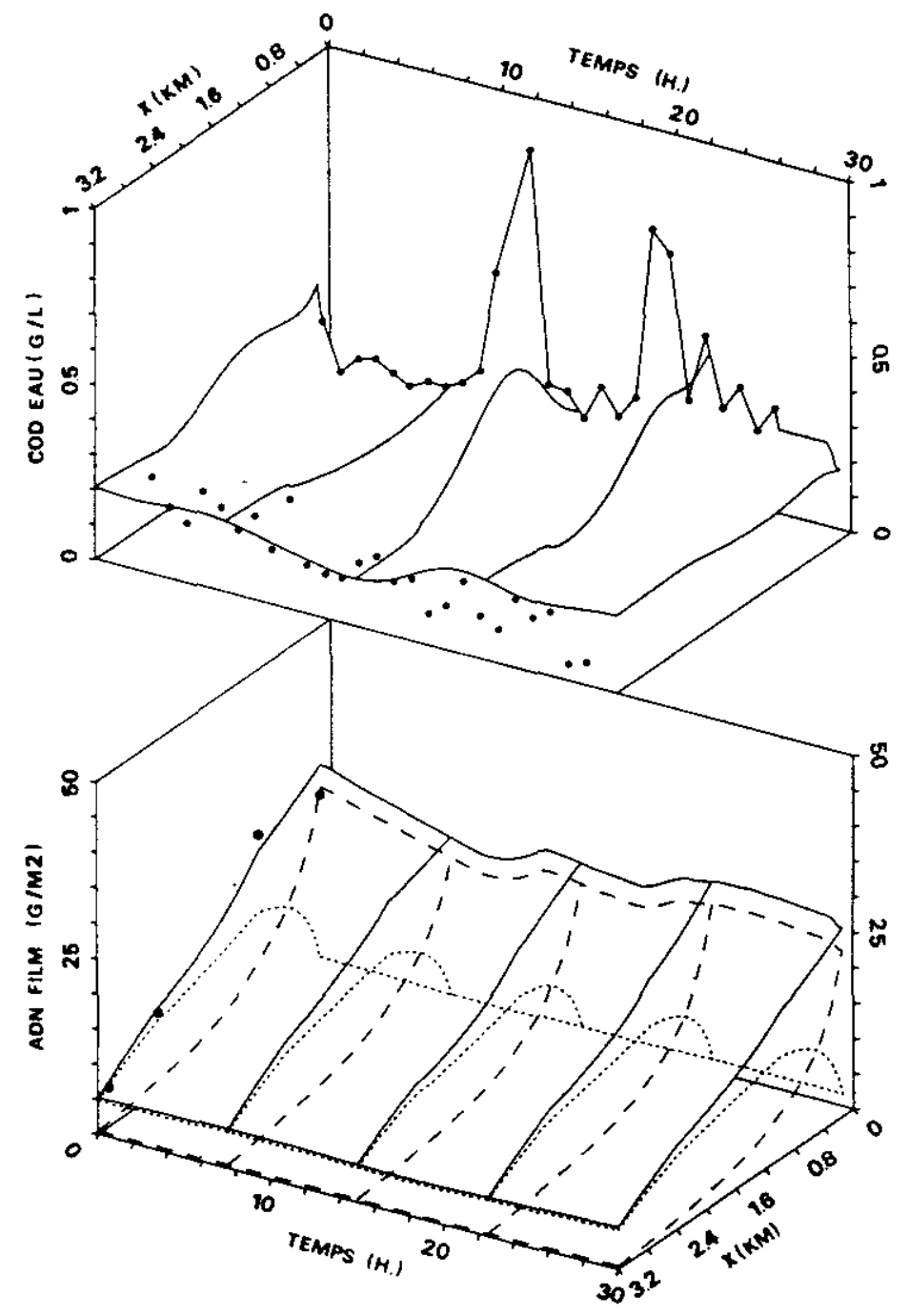

Figure 11. - Simulation de la dynamique du carbone organique dissous et de I'A.D.N. microbien benthique dans l'Albenche, utilisation d'un modẻle considérant deux populations ( $T \circ=$ 15.08 .83 à $22 \mathrm{~h}$ ) (* : valeurs expérimentales)

(- biomasse totale ; ----- biomasse "allochtone" ;

- - biomasse "saprophile").

Figure 11. - Simulation of the dissolved organic matter dynamics in the Albenche river (To $=15.08 .83$ at $22 \mathrm{~h}$ ) when two populatims of microorganisms are simulated (* : experimental values) :

- a allochtone population with low values for $\mu$ max. Ks and $\mathrm{Ke}$;

- a saprophile population with a hight affinity to organic wastes and high values for umax and $k s$ but with a low value for $\mathrm{Ke}$.

(-_- entire biomass ; -.--- "allochtone" biomass;

- . "saprophile" biomass). 
Les hypothèses qui ont permis la construction du modèle n'ont pas été mises en défaut par les simulations et les applications effectuées. Elles apparaissent donc cohérentes et soulignent deux points essentiels des processus par lesquels s'accomplit l'auto-épuration dans les petits cours d'eau :

- importance des mécanismes de transport (convection et dispersion longitudinale) ;

- prépondérance du compartiment benthique et des microorganismes fixés dans la dégradation des substrats organiques dissous.

De nombreuses lacunes et de nombreux problèmes restent en suspens. Mais, le modèle élaboré se révèle être un outil d'un grand intérêt tant sur le plan de la connaissance du système étudié que sur celui plus général des mécanismes d'auto-épuration de la matiēre organique dissoute dans les petits cours d'eau. Les informations ainsi obtenues devraient permettre des rétroactions sur l'établissement de plans de recherche pour continuer à progresser dans la compréhension de ces mécanismes.

Ce travail permet, également, de montrer les apports et les potentialités de la modélisation dans une telle étude.

\section{7 - NOTATIONS}

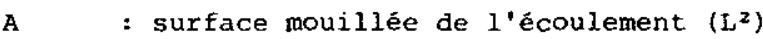

Af : surface d'une section de biofilm $\left(L^{2}\right)$

B : largeur de la section de l'écoulement, largeur au miroir (L)

$\mathrm{Ba}, \mathrm{Bai}$ : biomasse des macroinvertêbrēs benthiques (M. $\mathrm{L}^{-3}$ )

Bf, Bfi: biomasse bactérienne ou microbienne dans le biofilm benthique $\left(M \cdot I^{-2}\right)$

$\mathrm{Bi}$ : biomasse bactêrienne ou microbienne (M. $\mathrm{L}^{-3}$ )

Bis : biomasse bactérienne en suspension ( $\mathrm{M}^{-3} \mathrm{~L}^{-3}$ )

C : concentration en substrat organique (C.O.T. ou C.O.D.) (M. $\mathrm{L}^{-3}$ )

Cf : concentration en substrat organique dans le biofilm (M.L-3)

Cfo : concentration en substrat organique à la surface du biofilm (M. $\left.\mathrm{L}^{-3}\right)$

Cpbi : concentration en substrat organique particulaire dans le compartiment benthique (M. $\left.\mathrm{L}^{-2}\right)$

Cpsi : concentration en substrat organique particulaire en suspension (M. $\mathrm{L}^{-3}$ )

Cq : concentration en matière organique dissoute des apports (M. $\mathrm{L}^{-3}$ )

Cresp : respiration exprimée en carbone organique (M. $\left.\mathrm{L}^{-2} \cdot \mathrm{T}^{-1}\right)$

Df : coefficient de diffusion dans le biofilm $\left(L^{2} \cdot T^{-1}\right)$

$\mathrm{D}_{\mathrm{L}} \quad$ : coefficient de dispersion longitudinale $\left(\mathrm{L}^{2} \cdot \mathrm{T}^{-1}\right)$ 
ea, eai: coefficient de la relation dêbit-surface mouillée

$\mathrm{H}$ : hauteur d'eau (L)

$\mathrm{H}_{\text {Af }}$ : coefficient permettant le calcul de $\mathrm{Hf}\left(\mathrm{M}^{-1} . \mathrm{L}^{3}\right)$

Hf : épaisseur du biofilm (L)

Karr : taux d'arrachement du biofiln ('T

Ke : taux de consomation du substrat pour le métabolisme endogène $\left(\mathrm{T}^{-1}\right)$

Kresp : taux de respiration des microorganismes $\left(\mathrm{T}^{-1}\right)$

Ks : constante de demi-saturation pour les substrats organiques (M. $\mathrm{L}^{-3}$ )

Kt : vitesse de transfert à l'interface eau-biofilm (L.T $\mathrm{T}^{-1}$ )

$k_{2}, k_{2} i$ : taux de dégénérescence de la biomasse bactérienne ou microbienne $\left(\mathrm{T}^{-1}\right)$

Pm : pērimètre mouillé (L)

Q : débit $\left(L^{3} \cdot T^{-1}\right)$

$\mathrm{q}$ : dêbit d'apport $\left(\mathrm{L}^{3} \cdot \mathrm{L}^{-1} \cdot \mathrm{T}^{-1}\right)$

$\mathrm{T}$ : température $\left({ }^{\circ} \mathrm{C}\right)$

$t$ : temps (T)

$\mathrm{U} \quad$ : vitesse longitudinale moyenne (L. $\mathrm{T}^{-1}$ )

Up* : vitesse de frottement au fond $(\sqrt{g \cdot I \cdot R h})\left(L \cdot T^{-1}\right)$

xf : densité cellulaire dans le biofilm (biomasse bactérienne ou microbienne) (M. $\mathrm{L}^{-3}$ )

$x \quad$ : distance sur l'axe longitudinal (L)

$\mathrm{Y}_{1} \quad$ : coefficient de conversion exprimé en g de biomasse (g d'A.D.N.) édifiêe par $g$ de substrat ( $g$ de C.O.D.) utilisé

$\mathrm{Y}_{2} \quad$ : coefficient de relargage des cellules dêgénérées

$z \quad$ : épaisseur du biofilm (L)

$\alpha_{i} \quad$ : coefficient rentrant dans le calcul de BDI

BDL : coefficient rentrant dans le calcul de $D_{L}$

$\mathrm{Bi}$ : coefficient rentrant dans le calcul de BDL

$K_{\mathrm{pT}} \quad$ : paramètre $\mathrm{p}$ à la tempërature $\mathrm{T}$

umax : taux de croissance maximal $\left(\mathrm{T}^{-1}\right)$

$\theta$ : coefficient traduisant $l^{\prime}$ influence de la température

BECX M.B. (1983). A procedure for modeling. In : ORLOB G.T. (ed.), Nathematical modeling of water qualitu : streams, lakes, reservotis. Wiley, Chichester, p. $11-41$.
BOLING R.H., GOODMAN E.D., VAN SICKLE J.A., ZIMMER K.W., CUMMINS K.W., PETERSEN R.C., REICE S.R. (1975). Toward a model of detritus processing in a woodland stream. Ecology, 56 : 141-151. 
BOYLE J.D., SCOTT J.A. (1984). The role of benthic films in the oxygen balance in an east Devon river. Water Kes., 19 (9) : 1089-1099.

BUHR H.O., MILLER J.B. (1983). A dynamic model of the high-rate algalbacterial wastewater treatment pond. iater Res., 17 : 29-37.

CAUSSADE B., Chaussavotne C., Masbernat L. (1982). Modelling of turbulent fluxes in water surface ecosystems studies. Math. Comput. Sirmiation, 24 : 224-229.

CAzelles B. (1987). Modelisation d'un écosystème lotique : dynamique du carbone organique dissous et des microorganismes benthiques dans un cours d'eau pollué. Thèse de Doctoxat $n^{\circ} 6887$, Universitë Lyon I, $427 \mathrm{p}$.

CAZELLES B., FONIVIEILLE D. (1989). Modélisation d'un écosystème lotique pollué par whe charge organique : prise en compte de 1 'hydrodynamique et des mécanismes de transport. Se: soi. Jak, 2 = 183-209.

CHANG H.T., RITTMANN B.E. (I987a), Mathenatical modeling of biofilm on activated carbon. Environ. Sci. Tecinol., 21 : $273-280$.

CLESCERI L.S., PARK R.A., BLOMEIEtD J.A. (1977). General modei of microbial growth and decomposition in aquatic ecosystems. Appl. Environ. Aicrobiol., 33 : 1047-1058.

COSTERTON J.W., GEESEY G.G., CHENG K.J. (1978). How bacteria stick. Sci. Am., 238 (1) : 86-95.

EDELINE F. (1981a). Le pouvoir autoépurateur des rivières : recherche d'une equation globale. In DUBors D.M. (ed.), progress in ecological engineering and management by mathematical modelifing. Editions CEBEDOC. Liège, p. 151-182.

EDELINE F., BUET J.L. (1978). Nouvelles recherches sur la courbe en sac. Trib. $C E B E D E A U, 415: 273-280$.

EDELINE F., LAMBERT G. (1979). Selfpurification of rivers : a two-phase model for bacterial biodegradation. vater Res., 13 : 473-480.

FABRe J.. RAMANY bala P. (1980). Equations génêrales de transfert de masse, de quantitê de mouvemert, d'énergie, dans les eaux de surface. Ann. Univ. Abidjan, Sẽrie C. Tomes XVI : 70-102.

FonTVIEILLE D. (1978), Efficacité de 1 'auto-épuration et charge organique benthique le long d'un ruisseau pollué par une porcherie. Verh. Intermat. Verein. timet., 20: 1739-1735.
FONTVIEILLE D., CAZELLES B. (1985). MOdification de 1 importance relacive des flux de carbone organique d'u: ruisseau pollué aprés une période de crues. Yerh. Intermat. Verein. innol., 22 : 22702276.

FonTVietlle D., CAzetles $B$. (2988). Seasonal changes of some physiolceical and structural parameters in the sediments of an organically polluted stream. Verh. Intermat. Verein. Finnol., 23 : 1306-1312.

FONTVIEILLE D., EEVOTTE G. (1OS1). D.N.A. content of the sediment in relation to self-purification in streams polluted by organic wastes. :erh. Inteinst. "erein. Limmol., 21 (1) : 221-226.

FONTVIEILLE D., RENAUD $M$. (1982). A method of estimating the respiration of mud communities in shallow running water. water Res., 16 (5) : \$93-599.

FRY J.C. (1982). Interactions jetween bacteria and benthic invertebrztes. In: NEDWELL D.B. and BRCWN C.H. (eCs), Seaiment microbiology. Academic press, Loncon, p. 171-201.

GANTZER C.L., KOLLING H.P., RITTMANN B.E. LEWIS D.L. (1988). Predicting the rate of trace-organic compound removal by natural biofilms. Water Res., 22 (2) : 191200.

GATES W.E., MALAR J.T., WESTE:ELD J.D. (1969). The application of bacterial process kinetics in stream simulacion and stream analysis. Wajer Res., 3 : 663-686.

GEESY G.G., MUTCH R., COSTERTCN J.W., GREEN R.B. (1978). Sessile bacteria : an important component of the microbial population in small streams. : imol. Oceanogr. $23: 1214-1223$.

GENTIL S. (1982). Analyse de système et écologie : une étude de cas (Iac d'aiguebelette). In : aGUILAR-MARTIN $j$. (éd.), Modelisation mathermatique et simulation des systemes de l'environnemer.. Editions du C.N.R.S., Toulouse, p. 17-0̃.

HARREMOES P. (1982). Immediate and delayed oxygen depletion in rivers. ricter Res., 16 : 1093-1098.

HICKEY C.भ. (1988). Oxycen tizake kinetics and microbial biomass of river of sewage fungus biozilms. Water .es., 22 (11) : 1365-1373.

HVITVED-JACOBSEN T. (1982). The impact of combined sewer overflows on the dissolved owygen concentration of a river. water Res., 16 : 1099-1105. 
KAPLAN L.A., BCTT T.L. (1983). Microbial heterotroshic utilization of dissolved organic matter in a piedmont stream. Ereshwater Biology, 13 : 363377 .

KAPLAN L.A., BCTT T.L. (1985). Acclimatation of stream-bed heterotrophic microfloza : metabolic responses to dissolved organic matter. Freshuater Biology, 1 : 479-492.

KAWASHIMA H., SUzUKI M. (1989). Numerical simulation model for prediction of B.O.D. removal rate in streams. Water Sci. Technol., 21 : 1003-1014.

KARLSTROM U. (1978). Role of the organic layer on stones in detrical metabolism in streans. Verh. Intermat. Verein. Limnol., $20: 1463-1470$.

KISSEL J.C., Mac CARTY P.L., STREET R.L. (1984). Numerical simulation of mixedculture biofilm. d. Environ. Eng., 110 (2) : 393-411.

LA MOTTA J. (1976). Internal diffusion and reaction in biological films. Environ. Sci. Tecirrol., 10 (8) : 765-769.

LOCK M.A. (1981). River epilithon : a light and organic energy transducer. In : LOCK M.A. \& WILLIAMS D.D. (eds), Perspectives in muning water ecology. plenum Press, New-York, p. 3-40.

LOCK M.A., WALLACE R.R., COSTERTON J.W., VENTULLO R.M., CHARLTON S.E. (1984). River epilithon : toward a structuralfunctional model. Oikos, 42 : 10-22.

LIU H. (1977). Predicting dispersion coefficients of stream. J. Environ. Eng., $130: 56-69$.

MARTIN Y.P., LELONG P.P. (I981). Modélisation de la dynamique des communautés bactériennes d'un écosysteme planctonique marin expérimental. Ocecmologica Acta, $4(4): 433-443$.

Mac CALL P.L., FISHER J.B. (1980). Effects of tubificid oligochaetes on physical and chemical properties of lake Erié sediments. In : BRINKHURST R.O. \& COOK D.G. (eds), Aquatic Oligochaete biclogy, p. 253-317.

Mac INTIRE C.D. (1983). A conceptual framework for process lotic ecosystems. in : FONTAINE *.D. III \& BARTELL S.M. (eds), Dynamics of lotic ecosystems. Ann Arbor Science, Ann Arbor, p. 43-68.

MaC INTIRE C.D., COLBY 3.A. (1978). A hierarchical model of lotic ecosystems. Ecol. Honogr., 48 (2) : 167-190.
NEWBOLD J.D., ELWOOD J.W., O'NEIL R.V., SHELOON A.L. (1983). Phosphorus dynamics in a woodland stream ecosystem : a study of nutrient spiralling. Ecology, $64(5): 1249-1265$.

OHGAKI S., hWANG S.L., MatSUmCto J. (1976). Xinetics of attached microbial growth in a continuous stirred tank reactor. Water Res., 12 (4) : 243-249.

O'NEIL R.V., ELWDOO J.W., HILDEBRAND S.G. (1979a) Theoretical implications of spatial heterogeneity in stream erosystems. In : INNIS G.S. \& O'NEIL R.V. (eds), Systems analysis of ecosystems. International Cooperative Publishing House, p. 79-102.

O'NEIL R.V., EMANUEL W.R., NEWBOLD J.D., ELWOOD J.W. (1979b). Simulation in the service of environmental zesearch : experience with a stream ecosystem project. In : Sumer computer simulation conference proceedings. Simulation councils, La Jolla, California, p. 401-405.

RITTMANN B.E., Mac CARTY P.L. (1978). Variable order model of bacterial-film kinetics. J. Environ. Eng., 104 : 889900 .

RUTHERFORD J.C., O'SULEIVAN M.J. (1974). Simulation of water quality in Tarawera river. J. Environ. Eng., 100 (2) : 369390 .

SARNER E., MARKLUND S. (1985). Influence of particulate organics on the removal of dissolved organics in fixed-film biological reactors. Water Sci. mechnoi., $17: 15-26$.

SERVAIS P. (1987). Etude de la dégradation de la matière organique par les bactéries hétérotrophes en rivière. Développement $d$ 'une démarche méthodologique et application d la Meuse belge. These de Doctorat es Sciences, Université Libre de Bruxelles, $271 \mathrm{p}$.

SCHWARTZBROD J. (1985). Bactériologie des milieux aquatiques. Aspects écologiques et sanitaires. In : MARTIN G. (éd.), Point sur l'épuration et le traitement des effluents. 2.2 Bacteriologie des milieur cquatiques. Lavoișier, Paris, p. 47-113.

SPEITEL G.E., DOVANTZIS K., DI GIANO F.A. (1987). Mathematical modeling of biogeneration in G.A.C. columns. $J$. Inviron. Eng., 113 (1) : 32-48.

SIEGRIST H., GUJER w. (1985). Mass transfer mechanisms in a heterotrophic biofilm. Water Res., 19 (11) : 13691378 . 
SRINANTHAKUMAR S., AHIRTHARAJAH A. (1983). Organic carbon decay in stream with biofilm kinetics. J. Environ. Eng., 109 (1) = 109-119.

STEHFEST H. (1973). Mathematical modelling of self purification of rivers (in german ; english translation avaible as report IIASA PP 77 of the International Institute for applied Systems Analysis, Laxemburg. Austria. Report KFK 1654 UF Kernforschungszentrum. Karlszuhe West Germany.

STREETER H.W., PHELPS E.B. (1925). A study of the pollution and natural purification of the Onio Rivey. Public kealth Bulletin 145, U.S. Public Health Service, Washington $\mathrm{DC}$.

THERIEN N., MORRISON K.A., COUPAL B. (1981). The impact of reservoir flooding on a freshwater benthic comunity. In : MITSCH W.J., BOSSERMAN R.W. \& KLOPATEX J.M. (eds), Energy and ecological modelling. Elsevier, Amsterdam, p. 249-259.

TRULEAR M.G., CHARACKLIS W.G. (1982). Dynamics of biofilin processes. J. Water Pollut. Control Fed., 54 : 1288-1301.

VANDER BORGHT P., MICHEL D., WOLLAST R. (1981). Modélisation des déversements dans les petits cours d'eau : une étude de cas, la Semois. In : oubors D.M. (ed.), Progress in ecoiogisal engineering and management by mathematical modelting. Editions CEBEDOC, Liège, p. 517-537.
WANNER O.., GUJER W. (1985). Competition in biofilms. Water Sci. Teshnol., 17 : $27-44$.

WEBSTER J.R. (1983). The role of benthic macroinvertebrates in detritus dynamics of streams : a computer simulation. Ecol. Monogr., 53 (4) : 383404.

WEBSTER J.R., BENFIELD E.F., CAIRNS J. Jr (1979). Model predictions of the effects of impoundment on particulate organic matter transport in a river system. In : WARD J.V., STANFORD J.A. (eds), The ecology of regulated streams. Plenum Press, New-York, p. 339-364.

WILLIAMSON K.J., Mac CARTY P.L. (1976). A model of substrate utilisation by bacterial films. J. Water Poltut. Control Fed., $48: 9-24$.

WUHRMANN K. (1972) - Stream purification. In : MITCHELL R. (ed) : Water pollution misrobiology. Wiley, New-York, p. 1191.51 . 